\title{
An example of active circulation control of the unsteady separated flow past a semi-infinite plate
}

\author{
By L. CORTELEZZI, ${ }^{1} \dagger$ A. LEONARD ${ }^{2}$ AND J. C. DOYLE \\ ${ }^{1}$ Department of Engineering Science, California Institute of Technology, \\ Pasadena, CA 91125, USA \\ ${ }^{2}$ Graduate Aeronautical Laboratories, California Institute of Technology, \\ Pasadena, CA 91125, USA \\ ${ }^{3}$ Department of Electrical Engineering, California Institute of Technology, \\ Pasadena, CA 91125, USA
}

(Received 8 July 1992 and in revised form 26 July 1993)

Active circulation control of the two-dimensional unsteady separated flow past a semiinfinite plate with transverse motion is considered. The rolling-up of the separated shear layer is modelled by a point vortex whose time-dependent circulation is predicted by an unsteady Kutta condition. A suitable vortex shedding mechanism introduced. A control strategy able to maintain constant circulation when a vortex is present is derived. An exact solution for the nonlinear controller is then obtained. Dynamical systems analysis is used to explore the performance of the controlled system. The control strategy is applied to a class of flows and the results are discussed. A procedure to determine the position and the circulation of the vortex, knowing the velocity signature on the plate, is derived. Finally, a physical explanation of the control mechanism is presented.

\section{Introduction}

In recent years, the efforts to actively control unsteady separated fluid flows in a broad range of applications have become more intense, see Gad-el-Hak \& Bushnell (1991) for a discussion and references. In particular the control of the flow past bluff bodies is receiving a great amount of attention because of the large variety of applications: lift enhancement, drag reduction, noise and vibration control, mixing improvement, etc. Most of these studies are of an experimental nature while numerical contributions have increased in the past few years. Theoretical work is almost nonexistent, probably because of the high complexity of the equations governing the flow.

In approaching the problem of controlling a physical system, the choice of the mathematical model used to represent the system is of major importance: the selected model will influence the entire process of deriving and implementing the controller. If the system under investigation is a fluid flow the description through the full Navier-Stokes equations might be preferable for accuracy but would be dependent on large-scale computation and would not necessarily lead to any insights that would guide to the derivation of a control strategy. Consequently, the brute-force approach of controlling a full problem is unlikely to produce a controller which can be applied to an experimental situation in the near future. A different approach, the one taken in this study, is to derive a simpler fluid model which is able to capture the major features

$\dagger$ Present address : Department of Mathematics, University of California, Los Angeles, CA 900241555 USA. 
of the flow. With a simpler mathematical description one can hope to solve theoretically a class of interesting control problems and to produce fast numerical algorithms for control. Then, before attempting to control a laboratory flow, the controller derived for the simpler model might be used as a starting point to control the same class of flows simulated by a more sophisticated CFD code. The advantage of controlling a flow simulated by another CFD code is that it is possible to measure all the quantities required to feedback to the controller and, also, the action of the controller is naturally synchronized with the evolution of the flow because both algorithms run on virtual time. Furthermore, the use of higher-level simulator allows one to apply the derived controller to gradually more complicated situations. For example, if the controller were derived for an inviscid model, then it could be applied to the same flow when the fluid is slightly viscous. To perform properly in the new environment the controller has to be made robust about the new perturbation (see Doyle, Francis \& Tannenbaum 1992; Fan, Tits \& Doyle 1991). The iteration of the robustness process over different perturbations (e.g. viscosity, three-dimensionality, background noise, etc.) might produce a controller able to handle the flow modelled through the Navier-Stokes equations. The final ambitious step of actively controlling a real experiment might be undertaken only if the final product of this chain of refinements is a controller that is robust enough to perform in presence of the unmodelled and unpredictable uncertainties contained in the real system and which is 'fast' enough to control the flow in real time.

Recently, efforts to modify certain features of the wake behind bluff and slender bodies using open loop control, such as reduction or magnification of the wake thickness (Tokumaru \& Dimotakis 1991), vortex cancellation (Koochesfahani \& Dimotakis 1988), pattern reproduction (Ongoren \& Rockwell 1988a,b), and lift enhancement (Slomski \& Coleman 1993) have been successful. In all these investigations the free-stream velocity was kept constant and quasi-steady results were achieved usually by moving the body with a frequency scaled by the shedding frequency. In a more general situation in which the free-stream velocity is time dependent this approach is generally not sufficient to control the flow and a feedback control strategy is necessary.

In the present study we investigated active circulation control of a two-dimensional unsteady separated flow past a semi-infinite plate with transverse motion. As the authors have shown (Cortelezzi \& Leonard 1993), the motion of the plate affects sensibly the wake dynamics and it therefore should be useful for control purposes. Although extremely simple, this basic flow involves several important features common to many unsteady separation processes. In particular, because this flow can be interpreted as the two-dimensional unsteady analogue of the three-dimensional steady separation over a delta wing, it could provide deeper insight into vortex management concepts in such three-dimensional flows (Rao 1987). The aim of this paper is to show that, using a reasonably simplified model, it is mathematically possible to solve the control problem in a quite general way and to provide a complete framework in which to implement the feedback strategy. We model the unsteady separation from the transversely moving plate by a point vortex whose time-dependent circulation is predicted by an unsteady Kutta condition. This simplified model, first introduced by Brown \& Michael in 1954, is able to capture the main features of the flow and, for example, is quite accurate for power-law starting flows (see Rott 1956; Pullin 1978; Cortelezzi \& Leonard 1993). A suitable vortex shedding mechanism is also introduced to allow the simulation of flows involving multiple vortices. We then derive a control strategy which inhibits the production of further circulation when a vortex 
is present in the flow. Furthermore, we obtain for any time-dependent free-stream velocity of definite sign the analytical closed-form solution of the controller, i.e. the predicted motion of the plate that satisfies the Kutta condition without requiring further shedding of vorticity into the wake. The mathematical derivation of the controller shows that the fluid domain is divided in three controllability regions. The performance of the controller within these regions is then characterized by a dynamical systems type of analysis. The predictions of this analysis are confirmed by two numerical simulations in which the controller is successfully tested. Finally, we provide a procedure to determine the information necessary for feedback to the controller - the position and the circulation of the vortex - from the velocity signature on the plate, i.e. the velocity along the plate at the edge of the boundary layer. The analysis of the wall velocity shows that the signature is different depending on which controllability region the vortex is located. We conclude by presenting a physical argument that explains the control mechanism.

\section{Mathematical formulation}

In this section we present a mathematical model to represent two-dimensional unsteady separation from the tip of semi-infinite plate which is allowed to move transversely with respect to the unsteady free-stream velocity (Cortelezzi \& Leonard 1993). We restrict our investigation to the case where the shear layer that separates and is convected away is thin enough to justify a description by means of a vortex sheet. In addition we replace the spirals of the vortex sheet with point vortices. However, the vortex sheet is not completely lost; it is assumed to consist of a sheet of negligible circulation that connects the tip of the plate to a point vortex of variable strength which replaces the core of the forming spiral and satisfies an unsteady Kutta condition. Mathematically the feeding vortex sheet is just the branch cut due to the logarithmic singularity representing the vortex.

The frame of reference is fixed to the plate so that the body can be identified with the negative imaginary axis of the complex plane. Using Schwartz-Christoffel's technique we transform the semi-infinite plate in the $z$-plane onto the real axis in the $\zeta$-plane (see figure 1) with the following map:

$$
z=-\mathrm{i} \zeta^{2}
$$

We then build the complex potential $F$ by superimposing basic flows. Thus, the complex velocity field $w=\mathrm{d} F / \mathrm{d} \zeta$ in the mapped plane has the form

$$
w(\zeta, t)=U(t)+2 u_{p}(t) \zeta+\frac{\mathrm{i} \Gamma_{1}(t)}{2 \pi}\left(\frac{1}{\zeta-\zeta_{1}(t)}-\frac{1}{\zeta-\bar{\zeta}_{1}(t)}\right)+\sum_{n=2}^{N} \frac{\mathrm{i} \Gamma_{n}}{2 \pi}\left(\frac{1}{\zeta-\zeta_{n}(t)}-\frac{1}{\zeta-\bar{\zeta}_{n}(t)}\right) .
$$

Equation (2) contains the free-stream velocity, $U$, the velocity of the plate, $u_{p}$, and $N$ vortices of strength $\Gamma_{n}$ at $\zeta=\zeta_{n}$ with their images at the complex conjugate position $\zeta=\bar{\zeta}_{n}$. Note we use the convention that positive circulation corresponds to flow rotating in the clockwise sense. Note, that $U$ is labelled free-stream velocity for simplicity: in reality, it is the leading order of the expansion of the potential flow past a finite plate about the tip of the plate. We allow the strength of the first vortex to depend on time so that the Kutta condition may be satisfied. We choose the convention that the vortex of variable strength is labelled with the subscript 1 , so that whenever a vortex is shed all the others are renumbered. 

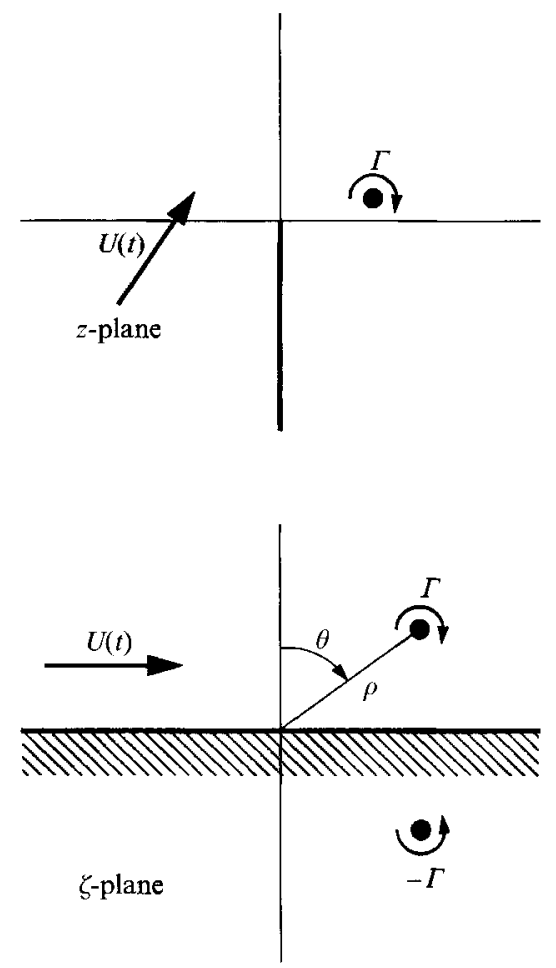

FIGURE 1. Physical and mapped planes.

We impose the Kutta condition to regularize the potential flow which, in the physical plane, presents a square-root singularity at the tip of the plate. To remove the singularity in the $z$-plane the complex velocity (2) in the mapped plane has to be zero at the origin all the time. Solving for $\Gamma_{1}$ we obtain

$$
\Gamma_{1}=2 \pi \mathrm{i}\left(\frac{\zeta_{1} \bar{\zeta}_{1}}{\zeta_{1}-\bar{\zeta}_{1}}\right)\left[U+\sum_{n=2}^{N} \frac{\mathrm{i} \Gamma_{n}}{2 \pi}\left(\frac{\zeta_{n}-\bar{\zeta}_{n}}{\zeta_{n} \bar{\zeta}_{n}}\right)\right]
$$

Note that $\Gamma_{1}$ does not depend directly on $u_{p}$ because the motion of the plate cannot affect the velocity of the fluid at the origin. In the mapped plane, in fact, the motion of the plate reduces to a stagnation-point flow about the origin. However, a change in the position of the plate modifies the relative position of the vortices with respect to the tip and so $\Gamma_{1}$ will be affected indirectly.

The motion of this system of vortices in the physical plane is described by the following set of ordinary differential equations:

$$
\begin{aligned}
& \frac{\mathrm{d} \bar{z}_{1}}{\mathrm{~d} t}+\left(\bar{z}_{1}-\bar{z}_{0}\right) \frac{1}{\Gamma_{1}} \frac{\mathrm{d} \Gamma_{1}}{\mathrm{~d} t}=\lim _{z \rightarrow z_{1}}\left\{\frac{\mathrm{d}}{\mathrm{d} z}\left[F-\frac{\mathrm{i} \Gamma_{1}}{2 \pi} \log \left(z-z_{1}\right)\right]\right\}, \\
& \frac{\mathrm{d} \bar{z}_{r}}{\mathrm{~d} t}=\lim _{z \rightarrow z_{r}}\left\{\frac{\mathrm{d}}{\mathrm{d} z}\left[F-\frac{\mathrm{i} \Gamma_{r}}{2 \pi} \log \left(z-z_{r}\right)\right]\right\},
\end{aligned}
$$

with the initial conditions

$$
z_{1}\left(t_{s}\right)=z_{0}, \quad z_{r}\left(t_{s}\right)=z_{r_{s}} \quad(r=2, \ldots, N)
$$

where $F$ is the complex potential and $z_{0}$ is the separation point. Note that $z_{r_{s}}$ is the final position of the vortex $r-1$ at the shedding time $t=t_{s}$, which becomes the initial 
position of the vortex $r$. Thus the number of equations increases every time a new vortex is shed. The term containing $\mathrm{d} \Gamma_{1} / \mathrm{d} t$ in $(4 a)$ is known as Brown \& Michael's correction (Brown \& Michael 1954). The motion of a vortex of variable strength described by this equation guarantees no net force on the vortex and its connecting cut. In the mapped plane the limiting process indicated in (4) produce the so-called Routh correction (e.g. Clements 1973).

For convenience we choose to solve the problem in the mapped plane. Once we have performed the change of variables, substituted for the complex potential and carried out the limits required in (4), we obtain

$$
\begin{aligned}
& {\left[2 \mathrm{i} \bar{\zeta}_{1}+\frac{\mathrm{i} \bar{\zeta}_{1} \bar{\zeta}_{1}}{\zeta_{1}-\bar{\zeta}_{1}}\right] \frac{\mathrm{d} \bar{\zeta}_{1}}{\mathrm{~d} t}-} {\left[\frac{\mathrm{i} \bar{\zeta}_{1}^{3}}{\zeta_{1}\left(\zeta_{1}-\bar{\zeta}_{1}\right)}\right] \frac{\mathrm{d} \zeta_{1}}{\mathrm{~d} t} } \\
&=\frac{\mathrm{i}}{2 \zeta_{1}}\left[U+2 u_{p} \zeta_{1}\right.\left.-\frac{\mathrm{i} \Gamma_{1}}{2 \pi\left(\zeta_{1}-\bar{\zeta}_{1}\right)}+\sum_{n=2}^{N} \frac{\mathrm{i} \Gamma_{n}}{2 \pi} \frac{\left(\zeta_{n}-\bar{\zeta}_{n}\right)}{\left(\zeta_{1}-\zeta_{n}\right)\left(\zeta_{1}-\bar{\zeta}_{n}\right)}-\frac{\mathrm{i} \Gamma_{1}}{4 \pi \zeta_{1}}\right] \\
&-\mathrm{i} \bar{\zeta}_{1}^{2}\left[\frac{\mathrm{d} U}{\mathrm{~d} t}+\sum_{n=2}^{N} \frac{\mathrm{i} \Gamma_{n}}{2 \pi}\left(\frac{1}{\zeta_{n}^{2}} \frac{\mathrm{d} \zeta_{n}}{\mathrm{~d} t}-\frac{1}{\bar{\zeta}_{n}^{2}} \frac{\mathrm{d} \bar{\zeta}_{n}}{\mathrm{~d} t}\right)\right]\left[U+\sum_{n=2}^{N} \frac{\mathrm{i} \Gamma_{n}}{2 \pi} \frac{\zeta_{n}-\bar{\zeta}_{n}}{\zeta_{n} \bar{\zeta}_{n}}\right]^{-1}, \\
& \frac{\mathrm{d} \bar{\zeta}_{r}}{\mathrm{~d} t}=\frac{1}{4 \zeta_{r} \bar{\zeta}_{r}}\left[U+2 u_{p} \zeta_{r}-\frac{\mathrm{i} \Gamma_{r}}{2 \pi\left(\zeta_{r}-\bar{\zeta}_{r}\right)}+\sum_{n \neq r}^{N} \frac{\mathrm{i} \Gamma_{n}}{2 \pi} \frac{\left(\zeta_{n}-\bar{\zeta}_{n}\right)}{\left(\zeta_{r}-\zeta_{n}\right)\left(\zeta_{r}-\bar{\zeta}_{n}\right)}-\frac{\mathrm{i} \Gamma_{r}}{4 \pi \zeta_{r}}\right],
\end{aligned}
$$

with the initial conditions

$$
\zeta_{1}\left(t_{s}\right)=0, \quad \zeta_{r}\left(t_{s}\right)=\zeta_{r_{s}} \quad(r=2, \ldots, N),
$$

where $\Gamma_{1}$ is given by (3). Note that because of Brown \& Michael's correction the equations are coupled not just through the position of all the vortices but also through their velocities.

The final element necessary for a correct implementation of this model is a vortex shedding mechanism. If we envision the separation process in terms of a vortex sheet then the instantaneous circulation necessary to keep the flow regular at the tip of the plate is associated with an infinitesimal segment of the sheet which is then shed into the fluid. The circulation is consequently distributed along the singular line and the sheet rolls up around the points of highest absolute circulation per unit length and it stretches most where the circulation per unit length is lowest. As the process continues, we observe that a large amount of circulation concentrates in the core of the spirals which are connected with each other by filaments of almost negligible circulation. This process can be reflected in our model by replacing each spiral with a point vortex of fixed circulation, except for the spiral connected to the separation point which is replaced by a point vortex with time-dependent circulation. This latter vortex will continue to be fed circulation until the rate of change of circulation becomes zero because we conjecture that this is the part of the sheet that will be stretched most.

Let us consider the problem of shedding a new vortex when $N-1$ other vortices are already present in the flow. If $t_{s}$ is the shedding time then it is crucial to analyse the transition from $t_{s}^{-}$to $t_{s}^{+}$. Up to the time $t_{s}^{-}$vortex 1 has variable strength such that the Kutta condition is satisfied. At time $t=t_{s}$ this vortex has its strength frozen and all the vortices are renumbered. Finally at $t_{s}^{+}$a new vortex 1 is introduced into the flow to remove the square-root singularity. If we restrict our simulation to the case where the shed vortices have alternate sign, then we can model the vortex shedding making the assumption that the time $t=t_{s}$ is determined by the condition:

$$
\left.\frac{\mathrm{d} \Gamma_{1}}{\mathrm{~d} t}\right|_{t=t_{s}}=0
$$


assuming $\mathrm{d}^{2} \Gamma_{1} /\left.\mathrm{d} t^{2}\right|_{t=t_{s}} \neq 0$. Any other choice for the shedding time implies the arbitrary production of two sequential vortices of the same sign or the existence of a vortex of strength that decreases in time. The latter situation is physically unacceptable. This procedure has been proposed by Graham (1980) to simulate the flow induced by an oscillating diamond-shaped cylinder.

The quality of the simulation with many vortices depends in large part on the shedding mechanism. Let us assume that between two zero crossings $\mathrm{d} \Gamma_{1} / \mathrm{d} t$ is positive and has two peaks, then it is not clear whether one or two vortices should be shed during this period. It seems that the deepness of the trough separating the peaks would be an important parameter. If it is very deep it seems reasonable to have two vortices, otherwise just one. Although the shedding mechanism can be implemented for all cases, to avoid any ambiguity we restrict ourselves to cases where the rate of circulation production has only one local maximum or minimum between consecutive zero crossings or, equivalently, where $\mathrm{d}^{2} \Gamma_{1} / \mathrm{d} t^{2}$ does not change sign between crossings.

\section{Active circulation control}

The authors have shown (Cortelezzi \& Leonard 1993) how the motion of the plate can modify the flow, in particular how it can affect the rate of circulation production. In the same work we derived the approximate solution of the equations of motion (6) which suggested that there may exist a particular $u_{p}(t)$ that inhibits the production of circulation. Now it seems legitimate to ask whether once the starting vortex has been shed, i.e. $t \geqslant t_{s}$, is it possible to move the plate in such a way that the Kutta condition remains satisfied without requiring a new vortex?

First let us recall the equations of motion in polar form for the starting vortex:

$$
\begin{aligned}
& \frac{\mathrm{d} \rho_{1}}{\mathrm{~d} t}=\frac{U \sin \theta_{1}}{12 \rho_{1}^{2}}-\frac{\rho_{1}}{3 U} \frac{\mathrm{d} U}{\mathrm{~d} t}-\frac{u_{p} \cos ^{2} \theta_{1}}{3 \rho_{1}}, \\
& \frac{\mathrm{d} \theta_{1}}{\mathrm{~d} t}=\frac{U \cos 2 \theta_{1}}{8 \rho_{1}^{3} \cos \theta_{1}}+\frac{u_{p} \sin 2 \theta_{1}}{2 \rho_{1}^{2}},
\end{aligned}
$$

with the initial conditions:

$$
\rho_{1}(0)=0, \quad \theta_{1}(0)=\theta_{0} .
$$

Note that the initial condition for $\theta_{1}$, i.e. the vortex departure angle, depends on the free-stream and plate velocities (see Cortelezzi \& Leonard 1993). Now if we assume, as we have before, that a reasonable criterion for vortex shedding is when the rate of circulation production goes to zero, i.e. $t=t_{s}$, then it is important to analyse the expression for $\mathrm{d} \Gamma_{1} / \mathrm{d} t$. The Kutta condition in polar form can be easily derived by the general expression for $\Gamma_{1}$, (3):

$$
\Gamma_{1}=\pi \frac{\rho_{1}}{\cos \theta_{1}} U .
$$

Taking the time derivative of (11) and using the equations of motion (9), we can write the rate of circulation production in terms of $U, \mathrm{~d} U / \mathrm{d} t$ and $u_{p}$ as follows:

$$
\frac{\mathrm{d} \Gamma_{1}}{\mathrm{~d} t}=\frac{\pi U}{3 \cos \theta_{1}}\left[U \frac{\left(8 \cos ^{2} \theta_{1}-3\right) \sin \theta_{1}}{8 \rho_{1}^{2} \cos ^{2} \theta_{1}}+\frac{2 \rho_{1}}{U} \frac{\mathrm{d} U}{\mathrm{~d} t}-u_{p} \frac{4 \cos ^{2} \theta_{1}-3}{\rho_{1}}\right] .
$$

It is interesting to observe that the plate velocity cannot affect the rate of circulation 
production if the angular position of the vortex is $\pm \frac{1}{6} \pi$. A more detailed analysis of this expression is presented in $\S 6$. Equating (12) to zero and solving for $u_{p}$ we have

$$
u_{p_{s}}=\frac{1}{4 \cos ^{2} \theta_{1_{s}}-3}\left[\frac{\left(8 \cos ^{2} \theta_{1_{s}}-3\right) \sin \theta_{1_{s}}}{8 \rho_{1_{s}} \cos ^{2} \theta_{1_{s}}} U_{s}+\left.\frac{2 \rho_{1_{s}}^{2}}{U_{s}} \frac{\mathrm{d} U}{\mathrm{~d} t}\right|_{t-t_{s}}\right] \text {. }
$$

Hence for this choice of $u_{p}$ the rate of circulation production is zero. At this point then we have a technique to discontinue feeding the starting vortex and to create a new vortex as long as $\theta_{1} \neq \pm \frac{1}{6} \pi$.

Let us assume for the moment that as long as one vortex of fixed circulation is present in the flow it is possible to move the plate in such a way that the Kutta condition is satisfied for all time. Then, from (4) this vortex of fixed strength $\Gamma_{1_{s}}$ moves in accordance with the following equations:

$$
\begin{aligned}
& \frac{\mathrm{d} \rho_{1}}{\mathrm{~d} t}=\frac{U \sin \theta_{1}}{4 \rho_{1}^{2}}-\frac{\Gamma_{1_{s}} \sin \theta_{1}}{16 \pi \rho_{1}^{3} \cos \theta_{1}}-\frac{u_{p} \cos 2 \theta_{1}}{2 \rho_{1}}, \\
& \frac{\mathrm{d} \theta_{1}}{\mathrm{~d} t}=\frac{U \cos \theta_{1}}{4 \rho_{1}^{3}}-\frac{\Gamma_{1_{s}}}{8 \pi \rho_{1}^{4}}+\frac{u_{p} \sin 2 \theta_{1}}{2 \rho_{1}^{2}},
\end{aligned}
$$

with the initial conditions:

$$
\rho_{1}\left(t_{s}\right)=\rho_{1 s}, \quad \theta_{1}\left(t_{s}\right)=\theta_{1 s},
$$

where $\rho_{1_{s}}, \theta_{1_{s}}$ and $\Gamma_{1_{s}}=\pi \rho_{1_{s}} U_{s} / \cos \theta_{1_{s}}$ are the values at the shedding time. From the Kutta condition or the requirement that the complex velocity be zero at the origin, we obtain the relationship

$$
\frac{\Gamma_{1_{s}}}{\pi} \frac{\cos \theta_{1}}{\rho_{1}}-U=0 .
$$

If this constraint on the trajectory of the vortex is satisfied for all time after the shedding time, $t_{s}$, then the Kutta condition is satisfied. We must now impose this constraint on the equations of motion (14). To do so we take the time derivative of (16) and we use the equations of motion (14) to rewrite the trajectory constraint in terms of $U, \mathrm{~d} U / \mathrm{d} t$ and $u_{p}$. Then solving for $u_{p}$ we obtain

$$
\begin{aligned}
u_{p}=\left[\frac{2 \pi^{2} \rho_{1}^{2} U^{2} \sin 2 \theta_{1}+\pi \rho_{1} U \Gamma_{1_{s}}\left(4 \cos ^{2} \theta_{1}-1\right) \sin \theta_{1}-\Gamma_{1_{s}}^{2} \sin 2 \theta_{1}}{8 \pi \rho_{1}^{2}\left(\pi \rho_{1} U \cos 2 \theta_{1}-\Gamma_{1_{s}} \sin 2 \theta_{1} \sin \theta_{1}\right) \cos \theta_{1}}\right. & \left.+\frac{2 \pi \rho_{1}^{3}}{\pi \rho_{1} U \cos 2 \theta_{1}-\Gamma_{1_{s}} \sin 2 \theta_{1} \sin \theta_{1}} \frac{\mathrm{d} U}{\mathrm{~d} t}\right] .
\end{aligned}
$$

This then is the speed that the plate has to assume, for $t \geqslant t_{s}$, to take advantage of the presence of the starting vortex now with constant circulation and keep the Kutta condition satisfied without forming a new vortex. The compatibility of the two parts of the argument can be verified by taking the limit of (17) for $t \rightarrow t_{s}$ and recovering the plate velocity (13). Substituting the plate velocity (17) in the equation of motion (14) we obtain

$$
\begin{aligned}
\frac{\mathrm{d} \rho_{1}}{\mathrm{~d} t} & =-\frac{\left(\Gamma_{1_{s}} \cos \theta_{1}-2 \pi \rho_{1} U\right) \Gamma_{1_{s}} \sin \theta_{1} \cos \theta_{1}-8 \pi^{2} \rho_{1}^{5}(\mathrm{~d} U / \mathrm{d} t) \cos 2 \theta_{1}}{8 \pi \rho_{1}^{3}\left(\Gamma_{1_{s}} \sin 2 \theta_{1} \sin \theta_{1}-\pi \rho_{1} U \cos 2 \theta_{1}\right)}, \\
\frac{\mathrm{d} \theta_{1}}{\mathrm{~d} t} & =\frac{\left(\Gamma_{1_{s}} \cos \theta_{1}-2 \pi \rho_{1} U\right) U \cos \theta_{1}-8 \pi \rho_{1}^{4}(\mathrm{~d} U / \mathrm{d} t) \sin 2 \theta_{1}}{8 \rho_{1}^{3}\left(\Gamma_{1_{s}} \sin 2 \theta_{1} \sin \theta_{1}-\pi \rho_{1} U \cos 2 \theta_{1}\right)}
\end{aligned}
$$


These are the equations of motion of the vortex in the controlled case. The trajectory of the vortex is affected by the displacement of the plate which keeps the Kutta condition satisfied.

Since the above equations implicitly satisfy the constraint (16), we can use such a relationship to decouple the equations of motion. After the simplification, (18) reduces to

$$
\frac{\mathrm{d} \theta_{1}}{\mathrm{~d} t}=\frac{1}{4 \cos ^{2} \theta_{1}-3}\left[\frac{\pi^{3}}{8 \Gamma_{1_{s}}^{3} \cos ^{2} \theta_{1}} U^{4}+\frac{\sin 2 \theta_{1}}{U} \frac{\mathrm{d} U}{\mathrm{~d} t}\right]
$$

with the initial condition

$$
\theta_{1}\left(t_{s}\right)=\theta_{1_{s}}
$$

where the radial position is

$$
\rho_{1}=\frac{\Gamma_{1_{s}}}{\pi} \frac{\cos \theta_{1}}{U}
$$

and the plate velocity (17) reduces to

$$
u_{p}=\frac{1}{4 \cos ^{2} \theta_{1}-3}\left[\frac{\pi\left(8 \cos ^{2} \theta_{1}-3\right) \sin \theta_{1}}{8 \Gamma_{1_{s}} \cos ^{3} \theta_{1}} U^{2}+\frac{2 \Gamma_{1_{s}}^{2} \cos ^{2} \theta_{1}}{\pi^{2} U^{3}} \frac{\mathrm{d} U}{\mathrm{~d} t}\right] .
$$

Analysing the denominator of (22) we can see that, in general, $\mathrm{d} \theta_{1} / \mathrm{d} t$ and $u_{p}$ become singular for

$$
U=0, \quad \theta_{1}= \pm \frac{1}{6} \pi, \pm \frac{1}{2} \pi .
$$

The constraint on $U$ means that it is not possible to reverse the direction of the free stream and at the same time maintain the Kutta condition without further production of circulation. In other words, when $U=0$ the plate prefers not to have a vortex in the flow because the singularity caused by the vortex cannot be balanced, hence the plate has infinite velocity, leaving the vortex behind. When $\theta_{1}= \pm \frac{1}{2} \pi$ the vortex touches the wall of the plate, and to avoid this situation the plate has to have an infinite speed. Finally, when $\theta_{1}= \pm \frac{1}{6} \pi$ the flow is uncontrollable because, in this case, the motion of the plate does not affect the rate of circulation production. Consequently, the physical plane is divided in three sectors of angle $\frac{2}{3} \pi$ each and the vortex cannot move from one to another without having the plate having infinite velocity.

The question asked at the beginning of this section has, hence, a positive answer. We derived the closed-form solution for the nonlinear controller that inhibits the production of circulation, for any given free-stream condition of definite sign, when the starting vortex is present in the flow. The controller predicts the plate velocity if, at any time, the free-stream velocity and acceleration and the vortex circulation and position are known. The present system might be classified as MISO, i.e. multiple-input singleoutput system. The problem of determining the position and the circulation of the vortex from the velocity distribution near the plate will be addressed in $\S 6$.

\section{Dynamical behaviour of the controlled system}

In the previous section we have been able to find a controller that inhibits the production of circulation when a vortex is present in the flow. Now, we are interested to know if it is possible to control the system for infinite time and, if so, how this might be done. If this is not possible, then we would like to understand how to optimize the performance of the controller. Hence, this section is devoted to the analysis of the dynamical behaviour of the controlled system.

We start by determining the fixed points of the unperturbed and uncontrolled system. The set of equations describing this case can be obtained by imposing $U(t)=U_{s}$ 
and $u_{p}(t)=u_{p_{s}}$ on (14). It is easy to prove that there does not exist any pair $\left(\rho_{1}, \theta_{1}\right)$ such that the right-hand sides of (14) are simultaneously zero. Physically this means that, given fixed free-stream and plate velocities, it is not possible to find a vortex of the necessary strength that at the same time does not move and satisfies the Kutta condition.

The lack of fixed points for the unperturbed system makes the search for periodic orbits of the perturbed one much more complicated. It also suggests the possibility of the total lack of such orbits. Note that the existence or lack of such orbits gives basic information about the possible performance of the controlled system. If a stable periodic orbit exists and if at the shedding time the vortex is located on the orbit then it will move on the orbit forever and consequently the system will be controllable for infinite time. On the other hand, if such orbits do not exist then it will be impossible to 'trap' the vortex and the control will eventually be lost. Because of the lack of fixed points the best way to conduct the search for periodic orbits is by investigating the global structure of the flow field.

The form of the equations of motion can be crucial for the analysis of the dynamics of the system. The equations for the perturbed and controlled case (18) can be simplified by the use of the trajectory constraint (16). The result of such simplification (19) is numerically very useful because it reduces the computation time and improves the accuracy but does not aid in the analysis. The right-hand side of (19) is singular, which violates the Lipschitz-continuity required to apply averaging methods. Also the use of the singular perturbation theory is very difficult in the case of periodic forcing, the one of interest, because at each cycle we have to match inner and outer solutions. A better way to use the trajectory constraint is to eliminate $\Gamma_{1_{s}}$ from (18). Substituting and simplifying we obtain

$$
\begin{aligned}
& \frac{\mathrm{d} \rho_{1}}{\mathrm{~d} t}=-\frac{1}{8 \rho_{1}^{2} U\left(4 \cos ^{2} \theta_{1}-3\right)}\left[U^{2} \sin \theta_{1}+8 \rho_{1}^{3} \frac{\mathrm{d} U}{\mathrm{~d} t} \cos 2 \theta_{1}\right], \\
& \frac{\mathrm{d} \theta_{1}}{\mathrm{~d} t}=\frac{1}{8 \rho_{1}^{3} U\left(4 \cos ^{2} \theta_{1}-3\right)}\left[U^{2} \cos \theta_{1}+8 \rho_{1}^{3} \frac{\mathrm{d} U}{\mathrm{~d} t} \sin 2 \theta_{1}\right] .
\end{aligned}
$$

Note that we recover the same singularities as defined by (23), but the right-hand sides present an interesting symmetry. Such a symmetry suggests the use of a Liapunov-type stability argument (e.g. Wiggins 1990) to determine the global behaviour of the system. In other words, we attempt to determine a function $V\left(\rho_{1}, \theta_{1}\right)$ such that its total derivative with respect to time has a definite sign for all pairs $\left(o_{1}, \theta_{1}\right)$. If such a function exists its level sets should provide global information about the evolution of the system. We limit the free-stream velocity so that it will not change sign because of the above singularity in $U$, and if we assume for the moment that $\theta_{1} \in\left[0, \frac{1}{2} \pi\right]$ or $\theta_{1} \in\left[-\frac{1}{2} \pi, 0\right]$ depending upon whether $U$ is positive or negative, then the free-stream acceleration is the only quantity that can change sign without constraint. Hence, the total derivative of $V$ with respect to time should not contain the term $\mathrm{d} U / \mathrm{d} t$. Helped by the symmetry of the equations we are able to find such a function:

$$
V=\rho_{1}^{2} \sin 2 \theta_{1}-A,
$$

which for $A \in[0, \infty)$ describes a family of hyperbolas which covers the entire first quadrant, i.e. $\forall \theta \in\left[0, \frac{1}{2} \pi\right]$. Taking the total derivative with respect to time we obtain

$$
U(t)>0, \quad \forall t>t_{s} \Rightarrow \frac{\mathrm{d} V}{\mathrm{~d} t}=\frac{\partial V}{\partial \rho_{1}} \frac{\mathrm{d} \rho_{1}}{\mathrm{~d} t}+\frac{\partial V}{\partial \theta_{1}} \frac{\mathrm{d} \theta_{1}}{\mathrm{~d} t}=\frac{U \cos \theta_{1}}{4 \rho_{1}}>0, \quad \forall t>t_{s} .
$$


The converse is also true. Strictly speaking the function $V$ cannot be called a Liapunov function because it does not have a definite sign (e.g. Wiggins 1990).

We assume from now on that $U(t)>0$ for all $t>t_{s}$. To interpret the above result let us assume that at time $t=t_{*}$ the position of the vortex is identified by the pair $\left(\rho_{1_{*}}, \theta_{1_{*}}\right)$; then the hyperbola that passes through this point is $V=\rho_{1}^{2} \sin 2 \theta_{1}-\rho_{1_{*}}^{2} \sin 2 \theta_{1_{*}}$. Now (26) proves that the vortex moves away from this curve to another with a higher value of $A$ and so on, i.e. the vortex drifts irreversibly away from the origin in this sense. This result gains greater physical meaning if we map the function $V$ onto the physical plane. Writing (25) in complex form and using the map (1) we obtain:

$$
V=\mathrm{i} \operatorname{Re}(z)-A \text {. }
$$

For $A \in[0, \infty)$ this function describes a family of straight vertical lines, parallel to the plate, which cover the entire right half-plane, i.e. $\forall x \geqslant 0$. Note that for $A=0$ the function $V$ coincides with the imaginary axis. Finally, we can conclude that the relationship (26) excludes the existence of any periodic orbit and consequently the controller cannot trap the vortex so that it remains near the plate, it may only slow down the drifting motion.

We can gain further insight into the behaviour of the controlled system by changing variables and describing the motion of the vortex in terms of a new pair $\left(A, \theta_{1}\right)$ where $\theta_{1}$ is the original angular variable and $A$ is defined as

$$
A=\rho_{1}^{2} \sin 2 \theta_{1} .
$$

Under this transformation the equations of motion (24) take the following form:

$$
\begin{aligned}
\frac{\mathrm{d} A}{\mathrm{~d} t} & =\frac{U\left(\sin \theta_{1} \cos ^{3} \theta_{1}\right)^{\frac{1}{2}}}{2(2 A)^{\frac{1}{2}}}, \\
\frac{\mathrm{d} \theta_{1}}{\mathrm{~d} t} & =\frac{U \sin 2 \theta_{1}}{4\left(2 A^{3}\right)^{\frac{1}{2}}\left(4 \cos ^{2} \theta_{1}-3\right)}\left[\left(\sin \theta_{1} \cos ^{3} \theta_{1}\right)^{\frac{1}{2}}+\frac{4\left(2 A^{3}\right)^{\frac{1}{2}}}{U^{2}} \frac{\mathrm{d} U}{\mathrm{~d} t}\right] .
\end{aligned}
$$

Note how (29a) basically restates the previous result (see (26)). Performing the same operation on (17) we obtain the following expression for the plate velocity:

$$
u_{p}=\frac{U}{2(2 A)^{\frac{1}{2}}\left(4 \cos ^{2} \theta_{1}-3\right) \sin 2 \theta_{1}}\left[\frac{\left(8 \cos ^{2} \theta_{1}-3\right)\left(\sin ^{5} \theta_{1}\right)^{\frac{1}{2}}}{\left(\cos \theta_{1}\right)^{\frac{1}{2}}}+\frac{4\left(2 A^{3}\right)^{\frac{1}{2}}}{U^{2}} \frac{\mathrm{d} U}{\mathrm{~d} t}\right]
$$

We now wish to understand how the singularity at $\theta_{1}=\frac{1}{6} \pi$ affects the evolution of the controlled system. The second term between square brackets in $(29 b)$ and (30) is common to both expressions. If we set

$$
P=\frac{4\left(2 A^{3}\right)^{\frac{1}{2}}}{U^{2}} \frac{\mathrm{d} U}{\mathrm{~d} t},
$$

then $P$ has the same sign as $\mathrm{d} U / \mathrm{d} t$. Using this term as parameter we can plot $\mathrm{d} \theta_{1} / \mathrm{d} t$ and $u_{p}$ versus $\theta_{1}$ (see figures 2 and 3 ). The presence of the singularity is clearly shown in both plots by the vertical line at $\theta_{1}=\frac{1}{6} \pi$ which divides the domain $\left(0, \frac{1}{2} \pi\right)$ in two parts. The plate velocity becomes infinite as the vortex approaches the values $\theta_{1}=0, \frac{1}{6} \pi, \frac{1}{2} \pi$, and consequently the motion of the vortex is confined in the sub-domains $\left(0, \frac{1}{6} \pi\right)$ or $\left(\frac{1}{6}, \frac{1}{2} \pi\right)$ depending on the initial conditions. Note how the behaviour of the singularity 
Active circulation control of unsteady separated flow

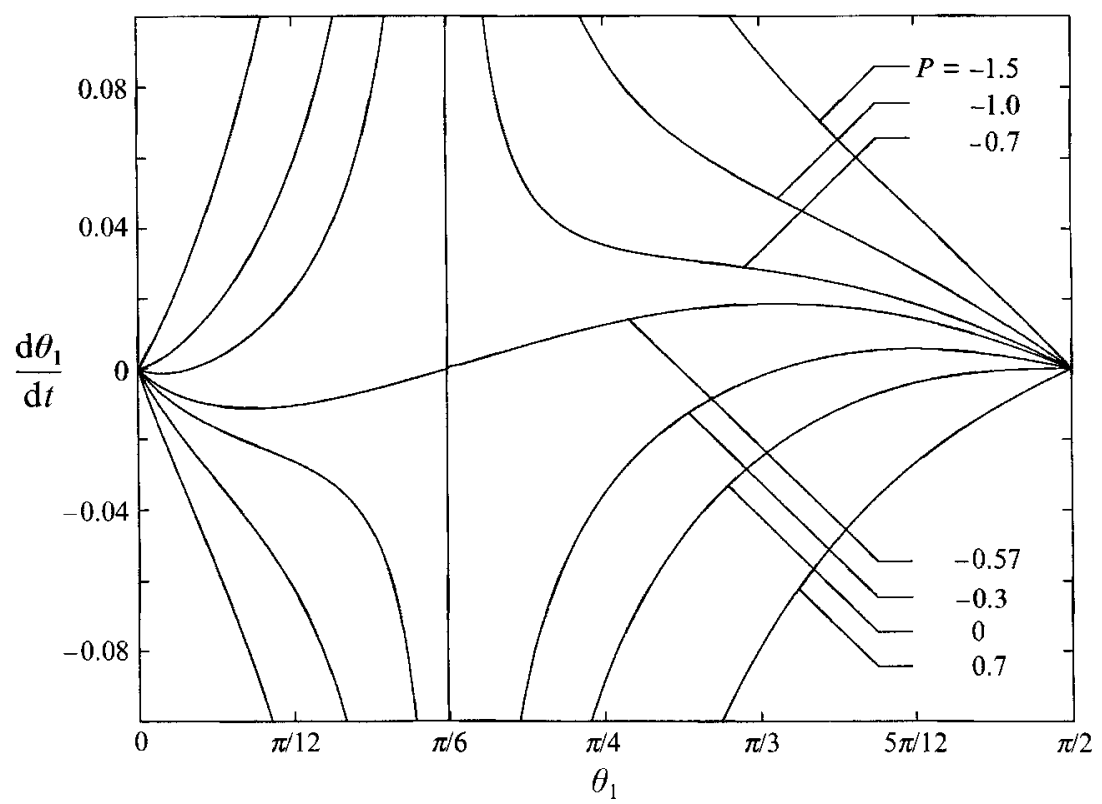

Figure 2. Qualitative behaviour of $\mathrm{d} \theta_{1} / \mathrm{d} t$ with respect to $\theta_{1}$.

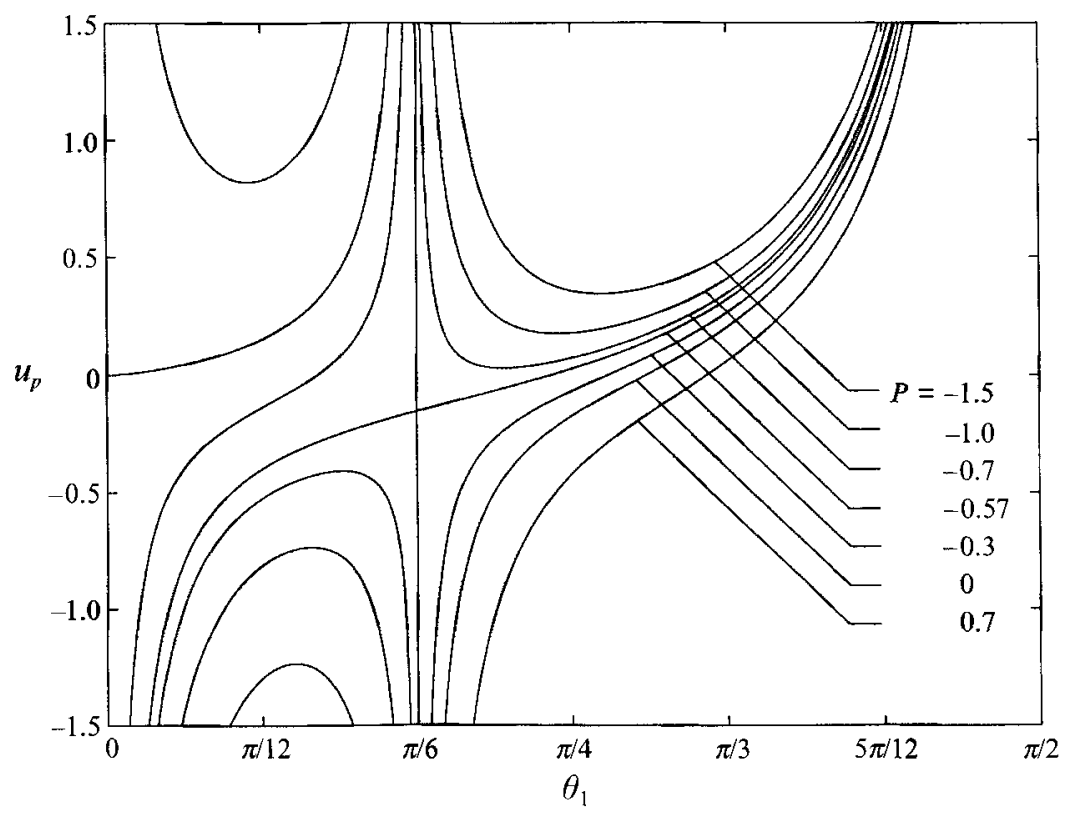

Figure 3. Qualitative behaviour of the plate velocity $u_{p}$ with respect to $\theta_{1}$.

depends on the sign and magnitude of $P$ : for $P>0$ the vortex is attracted by $\theta_{1}=\frac{1}{6} \pi$ while for $P<0$ it approaches or drifts away from the singularity depending on the initial condition and on the value of $P$. Figure 4 presents a phase portrait which summarizes such behaviour.

To understand better how the system evolves let us consider the vortex trajectories 


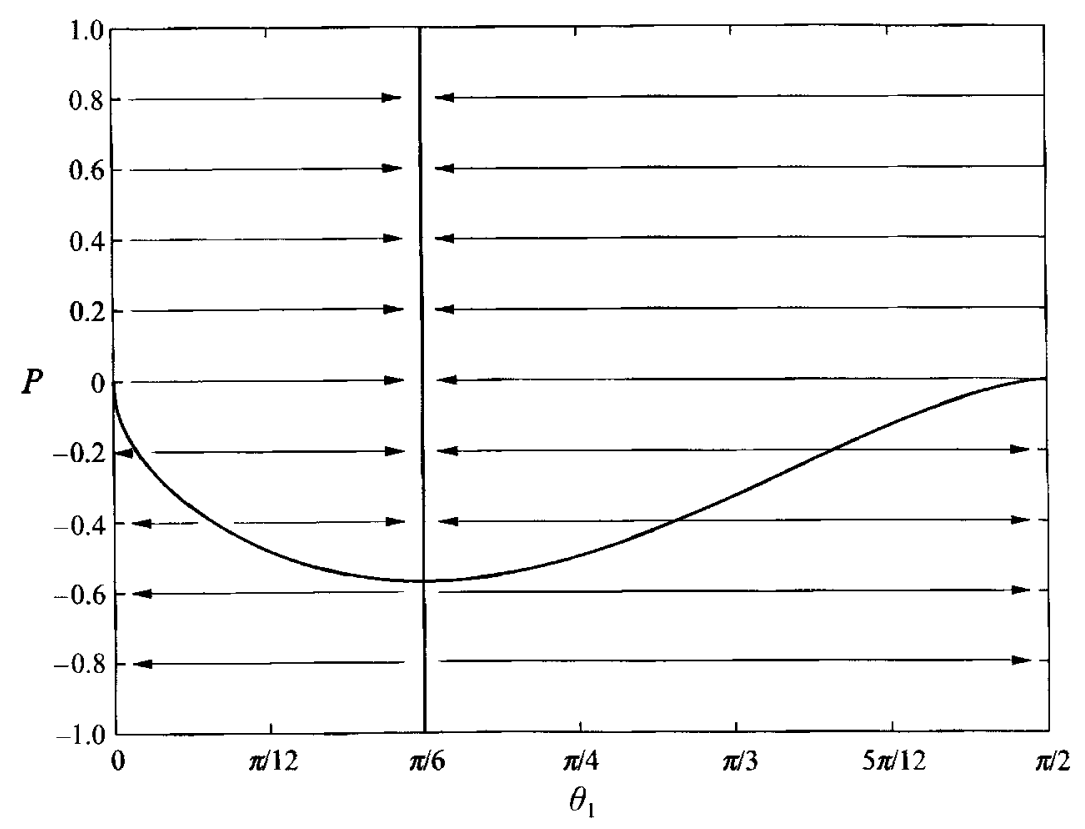

FIGURE 4. Phase portrait.

in some simple case. We obtain the equation for the vortex trajectory by taking the ratio of the equations of motion (24),

$$
\frac{\mathrm{d} \rho_{1}}{\mathrm{~d} \theta_{1}}=-\frac{1}{\rho_{1}}\left[\frac{U^{2} \sin \theta_{1}+8 \rho_{1}^{3}(\mathrm{~d} U / \mathrm{d} t) \cos 2 \theta_{1}}{U^{2} \cos \theta_{1}+8 \rho_{1}^{3}(\mathrm{~d} U / \mathrm{d} t) \sin 2 \theta_{1}}\right],
$$

with initial condition

$$
\rho_{1}\left(\theta_{1_{s}}\right)=\rho_{1_{s}} .
$$

This equation, in general, cannot be solved in closed form. However, for the particular choice

$$
\frac{1}{U^{2}} \frac{\mathrm{d} U}{\mathrm{~d} t}=B,
$$

where $B$ is a constant, an exact solution exists and the vortex trajectories are given in implicit form by the following expression:

$$
\frac{4 B \rho_{1}^{3} \sin 2 \theta_{1}-\cos \theta_{1}}{\rho_{1}}=\frac{4 B \rho_{1_{s}}^{3} \sin 2 \theta_{1_{s}}-\cos \theta_{1_{s}}}{\rho_{1_{s}}} .
$$

Let us analyse the following scenarios:

(i) $B=0 \Rightarrow \mathrm{d} U / \mathrm{d} t=0$

In this case the free-stream velocity is constant and the vortex trajectories, plotted in figure 5 , are described by the expression

$$
\rho_{1}=\rho_{1_{s}} \frac{\cos \theta_{1}}{\cos \theta_{1_{s}}}, \quad \forall t>t_{s} .
$$

Note that all the trajectories, independently of the initial condition, end up at $\theta_{1}=\frac{1}{6} \pi$. This is consequence of the fact that $P=0$ and hence the singular ray $\theta=\frac{1}{6} \pi$ behaves 


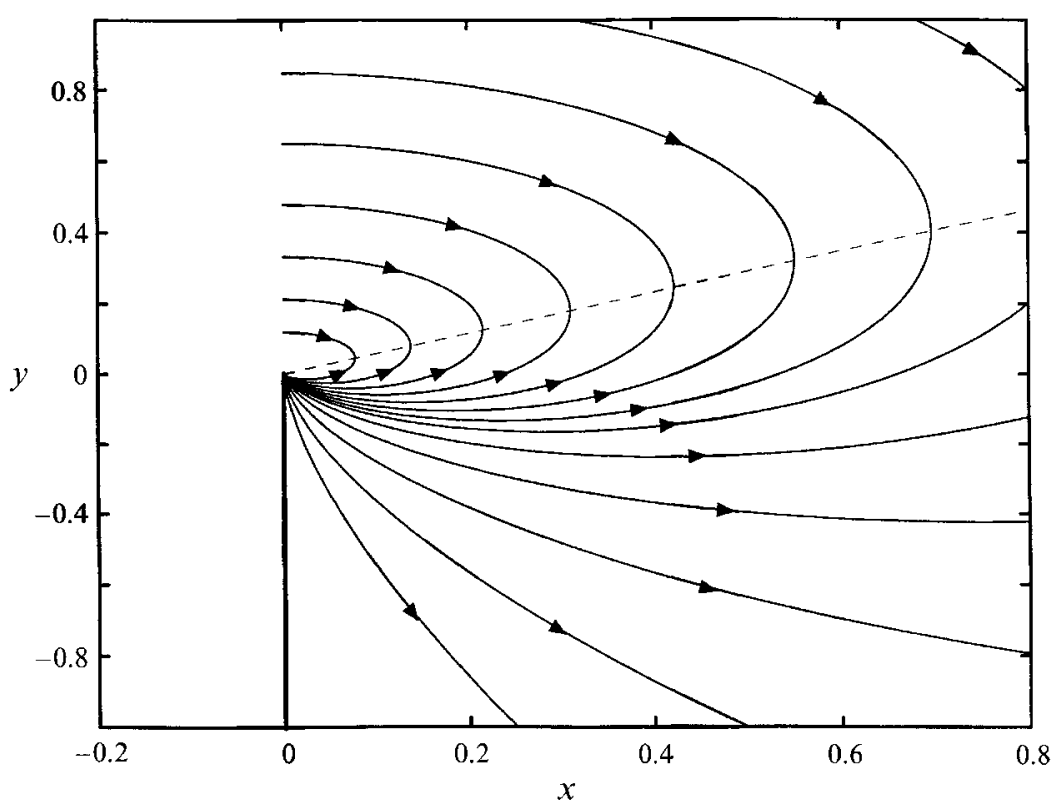

FIGURE 5. Vortex trajectories $(U(t)=$ const. $)$.

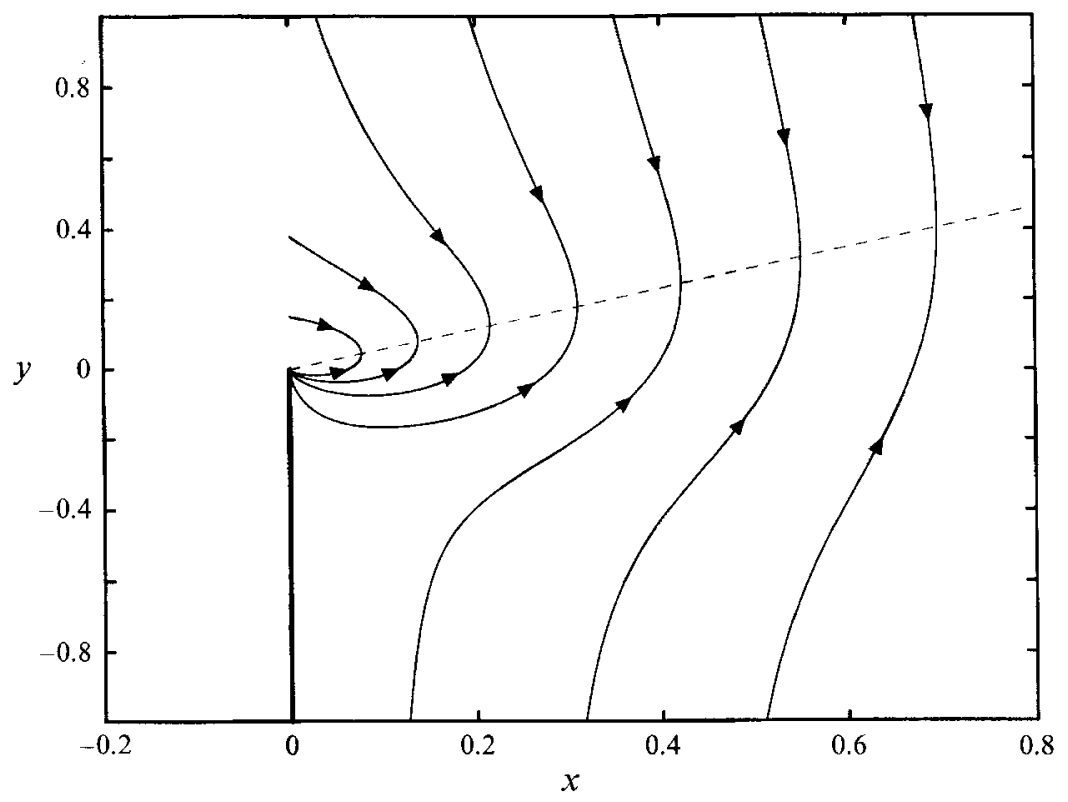

FIGURE 6. Vortex trajectories $\left(\mathrm{d} U / \mathrm{d} t=U^{2}\right)$.

as an attractor (see figure 4). As the vortex moves along one of these trajectories the plate picks up speed, which tends to infinity as the vortex approaches the singularity (see figure 3). 


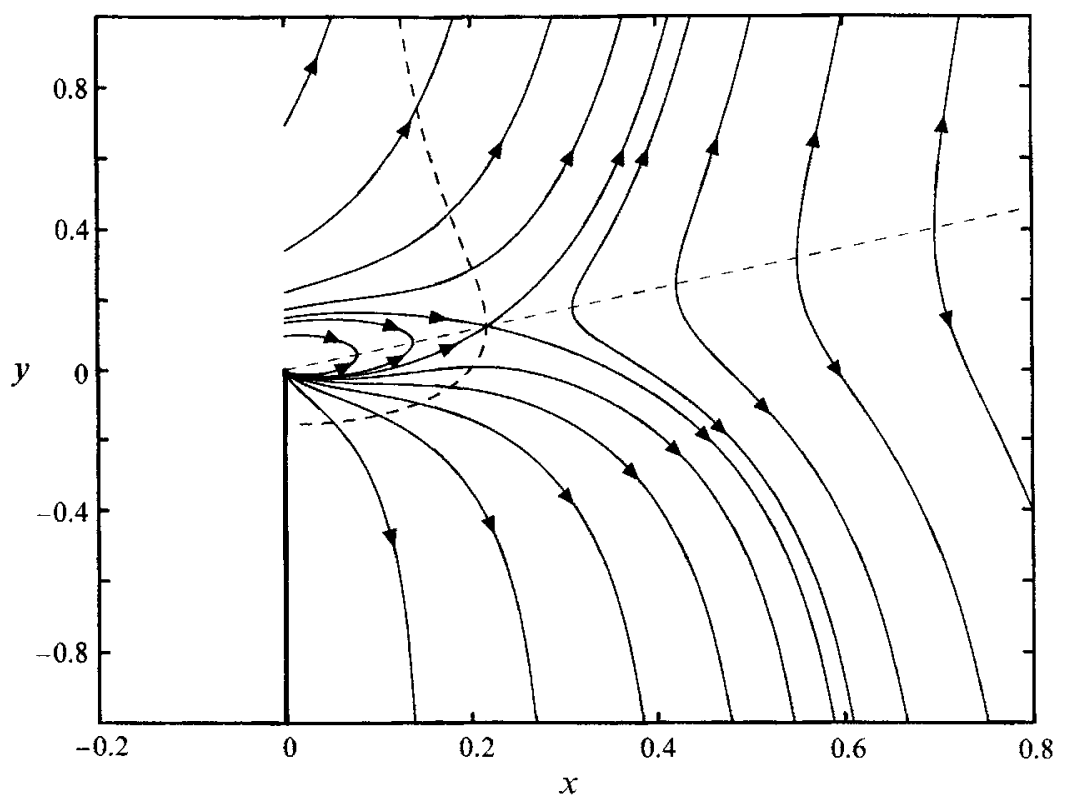

FIGURE 7. Vortex trajectories $\left(\mathrm{d} U / \mathrm{d} t=-U^{2}\right)$.

(ii) $B>0 \Rightarrow \mathrm{d} U / \mathrm{d} t>0$

In this case the free-stream velocity increases monotonically and has the form

$$
U(t)=\frac{U_{s}}{1-B U_{s}\left(t-t_{s}\right)}, \quad \forall B>0, \quad \forall t \in\left(t_{s}, t_{s}+\frac{1}{B U_{s}}\right) .
$$

Figure 6 shows the vortex trajectories (35) for this case. As in the previous case all the curves, independently of the initial condition, end up at $\theta_{1}=\frac{1}{6} \pi$. The singularity behaves, again, as an attractor because $P>0$ (see figure 4), but its strength is greater than before (see figure 2), explaining why the trajectories approach the singularity more directly. The plate velocity has the same trend as before but its magnitude is larger (see figure 3).

(iii) $B<0 \Rightarrow \mathrm{d} U / \mathrm{d} t<0$

In this case the free-stream velocity decreases monotonically and has the form

$$
U(t)=\frac{U_{s}}{1-B U_{s}\left(t-t_{s}\right)}, \quad \forall B<0, \quad \forall t>t_{s} .
$$

The trajectories (35) are plotted in figure 7 . The complexity of the pattern is because the singularity at $\theta_{1}=\frac{1}{6} \pi$ behaves as an attractor or as a repulsor depending on the vortex position and on the value of $P$. The dashed curve in figure 7 is the solution of the following equation:

$$
\left(\sin \theta_{1} \cos ^{3} \theta_{1}\right)^{\frac{1}{2}}+P=0,
$$

which determines when $\mathrm{d} \theta_{1} / \mathrm{d} t$ changes sign (see (29)). When the vortex moves within the area defined by the imaginary axis and the dashed line its angular position is attracted by the singularity. Outside this region the singularity switches behaviour and the vortex drifts away from the dashed line $\theta_{1}=\frac{1}{6} \pi$. Note there are trajectories that 
cross the dashed curve. In these cases the attractor is not strong enough to keep the vortex inside the region. Thus when it crosses the dashed curve the singularity starts to repel the vortex, which moves irreversibly away.

(iv) $\mathrm{d} U / \mathrm{d} t=\sin (\omega t+\phi)$

This is a case of simple periodic forcing: an exact solution is not available but in each period we satisfy the condition of one or other of the previous two cases. Note that the upper bound on the acceleration amplitude is given by the free-stream magnitude which we restrict not to go to zero nor change sign. In this case a comprehensive quantitative analysis is possible using the Poincaré map. As an alternative to this computationally intensive technique the evolution of the system will be described qualitatively. The parameter $\boldsymbol{P}$ swings from positive to negative values in accord with the free-stream acceleration and consequently the singularity acts as an attractor or as a repulsor in agreement with it (see figure 4). It is important to observe that over an entire period the singularity behaves as an attractor for more than half a period, hence we speculate that the vortex drifts downstream, fatally attracted by it. Figures 2 and 3 suggest that the evolution of the system is largely affected by the initial condition and that an escape to infinity is still possible but unlikely.

From the above analysis it follows that the performance of the controller can be greatly improved by an appropriate choice of the initial condition. Since the vortex eventually ends up on the border of the controllability region, i.e. on $\theta_{1}=\frac{1}{6} \pi$, then the best initial condition is the one that forces the time necessary to reach the singularity to be as large as possible. This time can be formally expressed as

$$
t_{t o t}=\int_{\theta_{s}}^{\frac{1}{6} \pi}\left(\frac{\mathrm{d} t}{\mathrm{~d} \theta_{1}}\right) \mathrm{d} \theta_{1}
$$

Then, it would seem that $t_{\text {tot }}$ can be made larger by choosing the initial condition so that the integrand is singular at $t=t_{s}$. It follows that the best $\theta_{1_{s}}$ is the one for which

$$
\left.\frac{\mathrm{d} \theta_{1}}{\mathrm{~d} t}\right|_{t=t_{s}}=0
$$

and consequently

$$
\left(\sin \theta_{1_{s}} \cos ^{3} \theta_{1_{s}}\right)^{\frac{1}{2}}+P\left(t_{s}\right)=0 .
$$

Note that (42) has a solution only if $-(27)^{\frac{1}{4}} / 4<P<0$, i.e. when the free stream decelerates. In the case of uncontrolled periodic forcing it is possible to prove that the first vortex is cut away when $\mathrm{d} U / \mathrm{d} t<0$ (see Cortelezzi 1993), hence the optimal $\theta_{1_{s}}$ might be determined.

\section{Results}

In Cortelezzi \& Leonard (1993) we presented a simulation where the free stream at time zero suddenly rose and then oscillated about a non-zero mean. We chose such a free-stream variation because it closely resembles that associated with the flow past a plate that is put into flow-induced vibrational motion. In that case we did not move the plate transversely and a sequence of vortices was produced. We now use the same freestream oscillations but move the plate to inhibit the production of circulation. We present two cases, one in which the motion of the vortex is confined in the third sector 


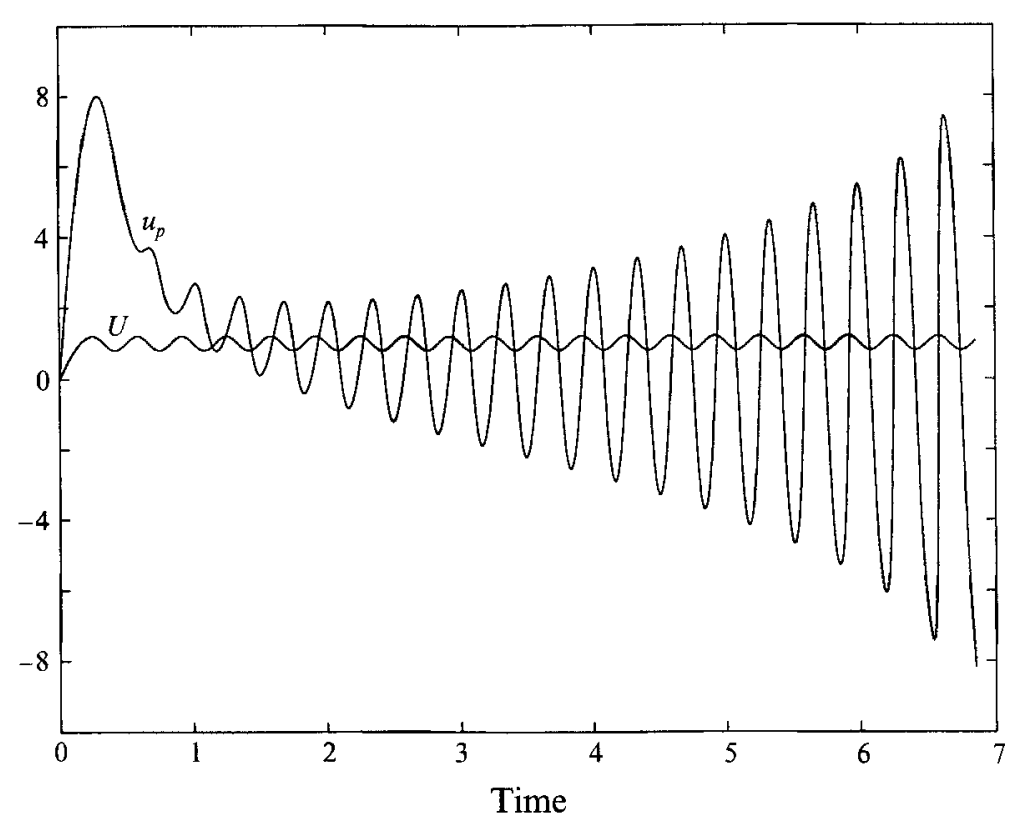

FIGURE 8. Free-stream and plate velocities.

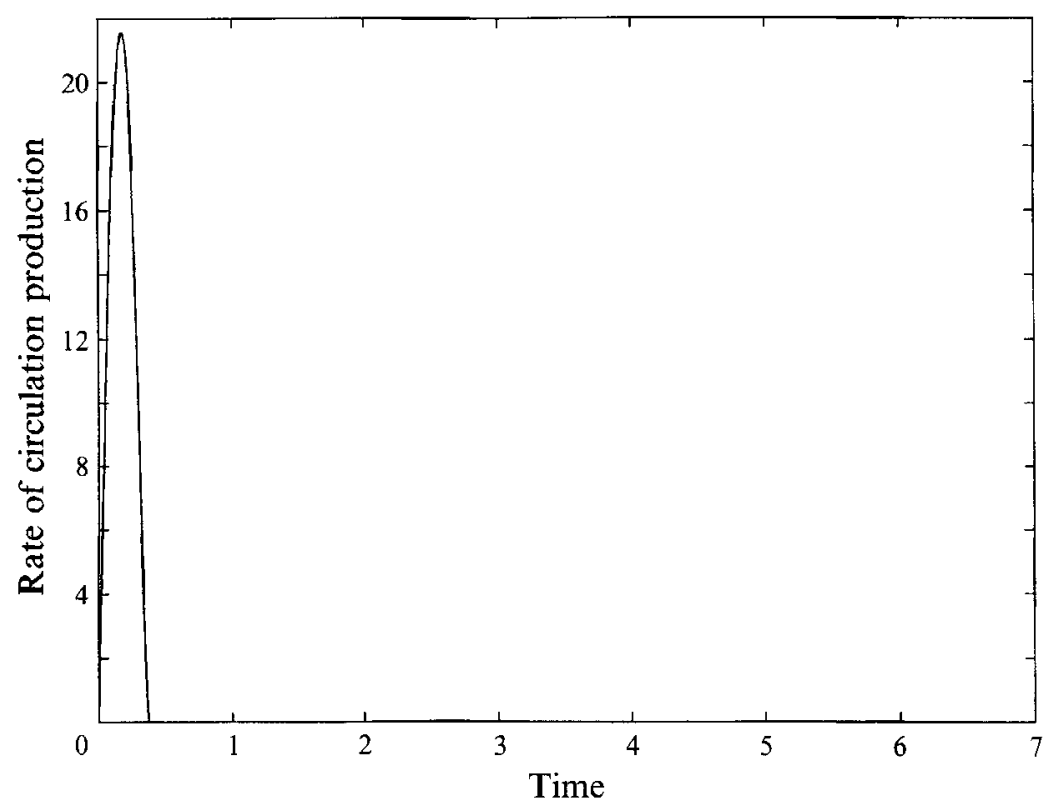

FIGURE 9. Rate of circulation production.

and another where the vortex stays within the second. As we have noticed previously the formulation (19)-(22) is the most convenient for a numerical integration.

We start active circulation control when the vortex is in the third sector because in the uncontrolled case (see Cortelezzi \& Leonard 1993) the first vortex is naturally shed in this region. Figures $8-12$ illustrate the growth of the starting vortex and the evolution of the controlled system. The velocity of the plate up to the shedding time 


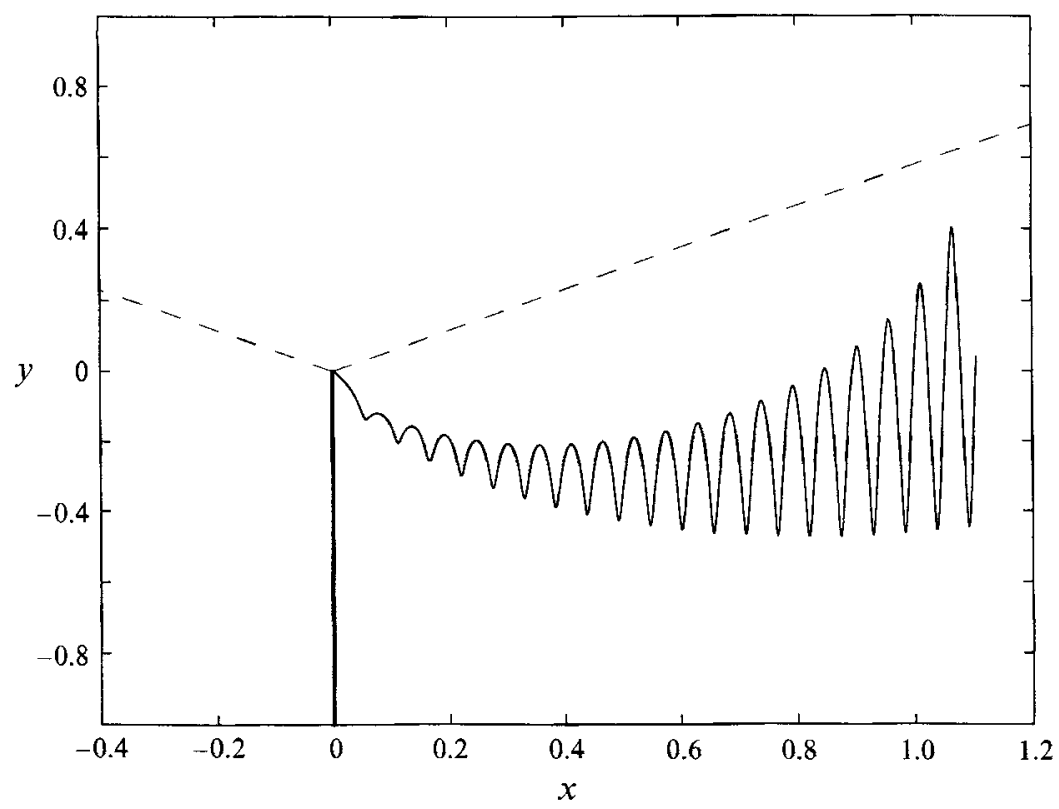

FigURE 10. Starting vortex trajectory.

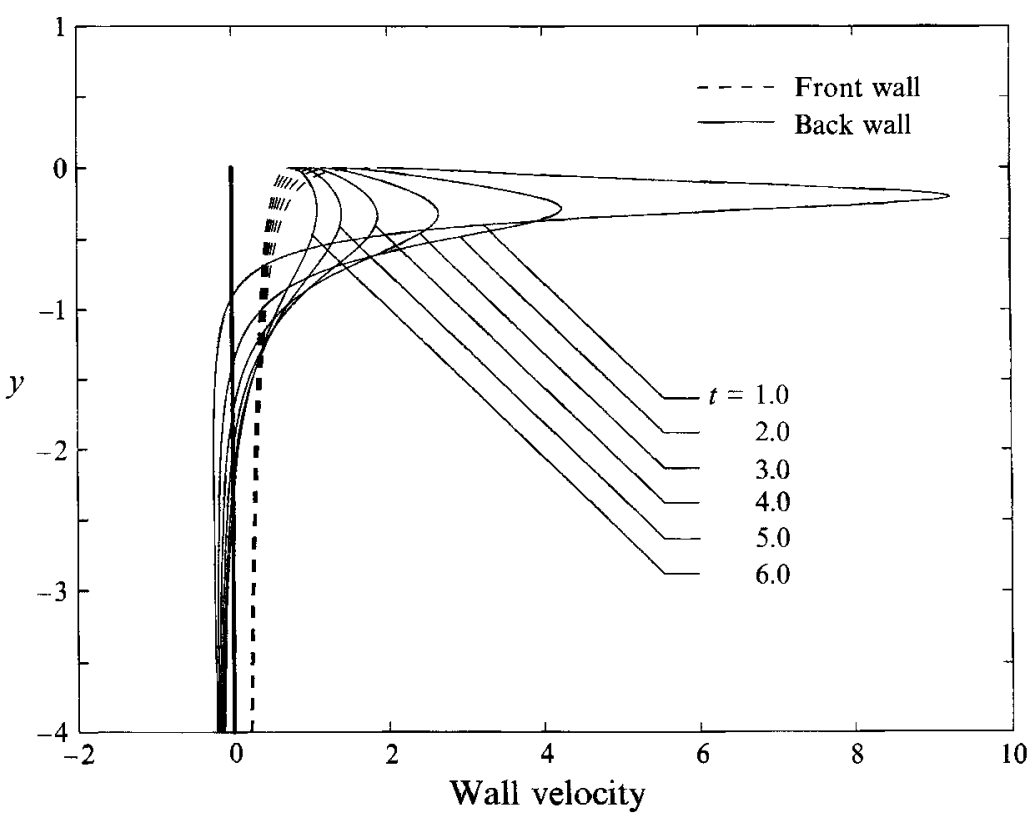

FIGURE 11. Velocity signature on the walls of the plate when $u_{p}$ is subtracted out.

has been chosen to provide a reasonable distance between the vortex location and the sector divider. Figure 9 shows that up to time $t_{s} \approx 0.32$ the starting vortex grows in circulation. When the rate of circulation production goes to zero the strength of the vortex is frozen, triggering active control for the rest of the simulation $\left(t_{\text {final }} \approx 6.75\right)$. From figures 8 and 10 we can see that as the vortex is convected farther downstream the plate velocity must increase to maintain control. Also, as the trajectory becomes 
(a)

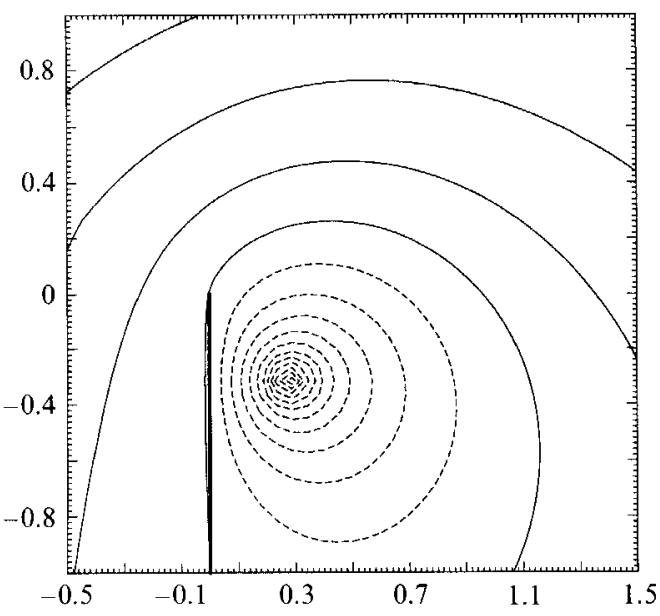

(c)

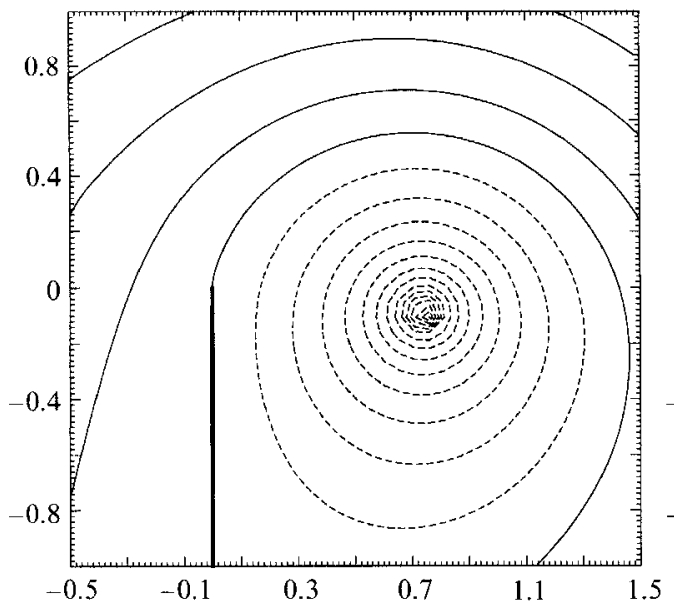

(b)

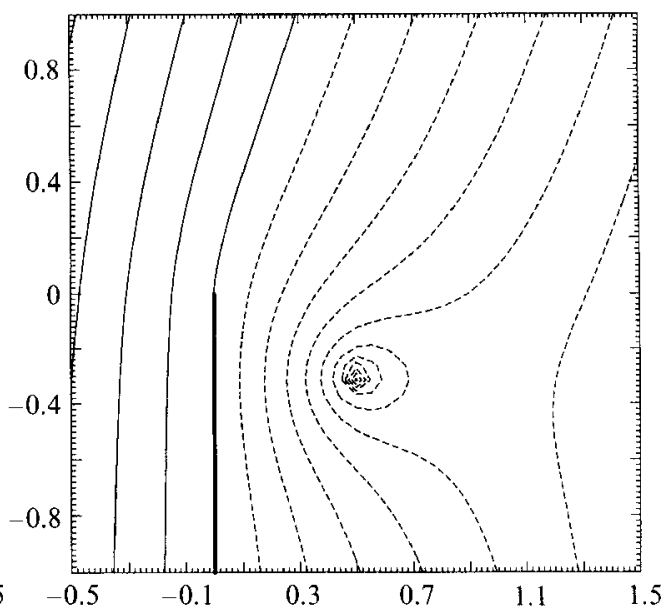

(d)

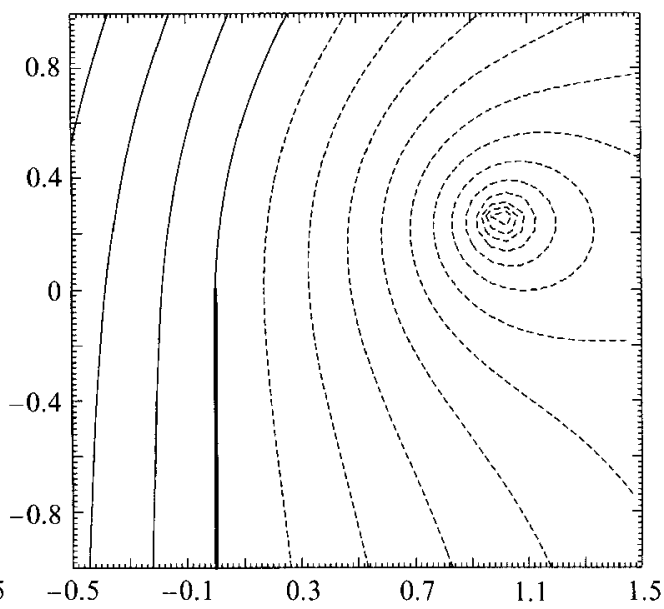

Figure 12. Shedding control: (a) $t=1.79,(b) t=3.17$, (c) $t=4.59,(d) t=6.25$.

closer to the sector divider the speed of the plate becomes larger making the situation progressively more critical with a complete loss of control for $t>t_{\text {final }}$. Figure $12(a d)$ shows the instantaneous streamlines during the controlled period, giving insight into the behaviour of the flow near the tip of the plate.

An example of active shedding control within the second sector is given by figures 13-17. The free-stream velocity is the same as before and the plate velocity up to the shedding time is basically the same but with opposite sign to provide the necessary initial condition. In this case the circulation of the starting vortex becomes constant at time $t_{s} \approx 0.35$, see figure 14 . Figures 13 and 15 show that as soon as the controller takes over it imposes large oscillations on the plate motion, but the amplitude grows only slowly in time. Roughly speaking the plate moves as close to or as far away from the vortex as is necessary to maintain the Kutta condition. Figure $17(a-d)$ shows the instantaneous streamlines during the controlled period.

The different behaviour of the controller in the two cases is a direct consequence of the position and strength of the vortex at the shedding time. In the first case (vortex 


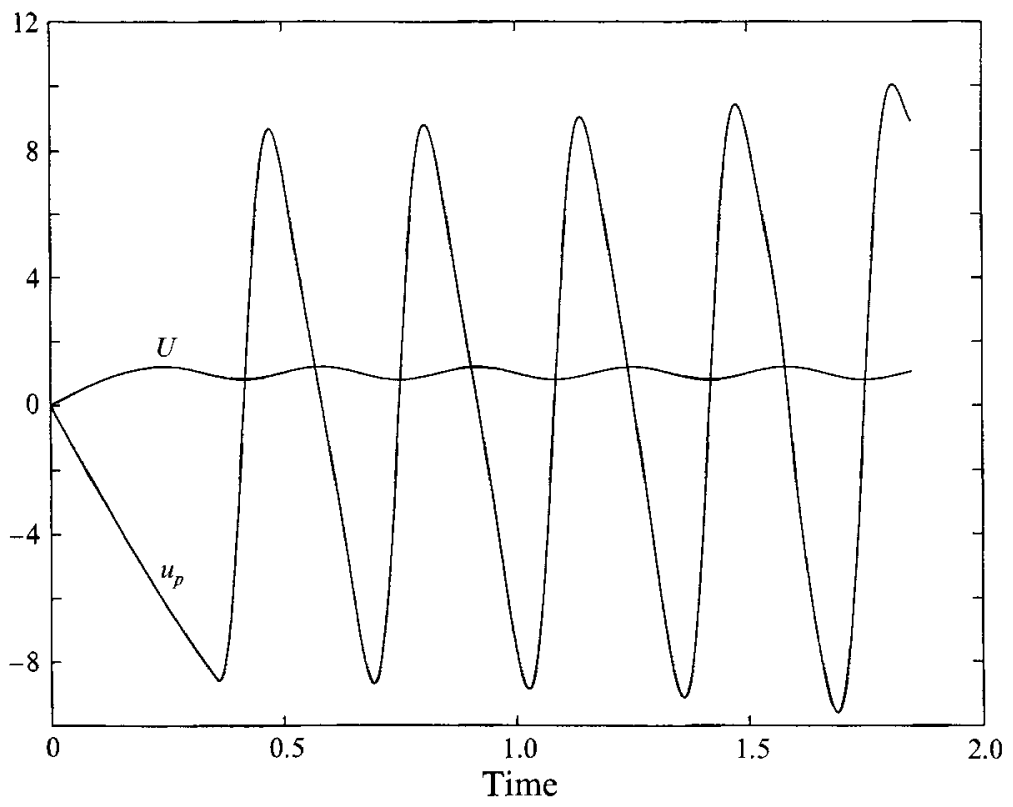

FiguRE 13. Free-stream and plate velocities.

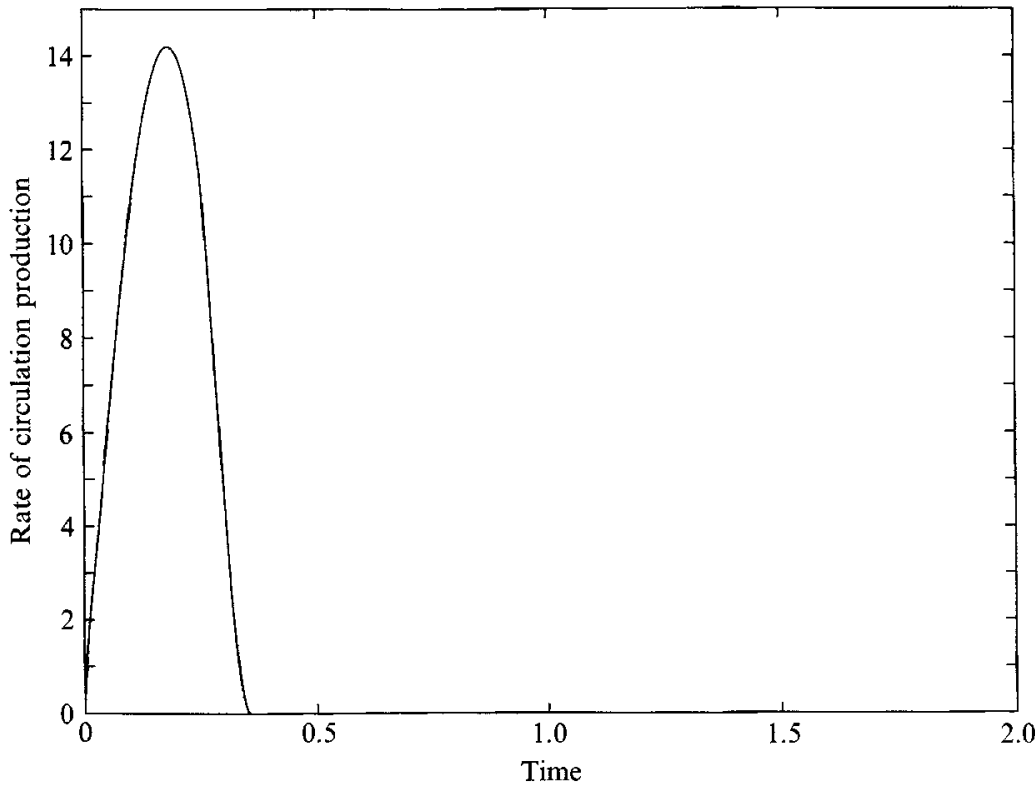

FIGURE 14. Rate of circulation production.

in the third sector) the plate at the beginning is moving into the flow and a strong vortex is trapped in the recirculating bubble near to the leading edge. Initially, slight movements of the plate are enough to satisfy the Kutta condition, then as the vortex drifts downstream larger oscillations are required. Of great help in this case is the fluid flowing around the edge which 'keeps' the vortex away from the attractor and the vortex strength which maintains smooth flow even if the vortex is quite far downstream. 


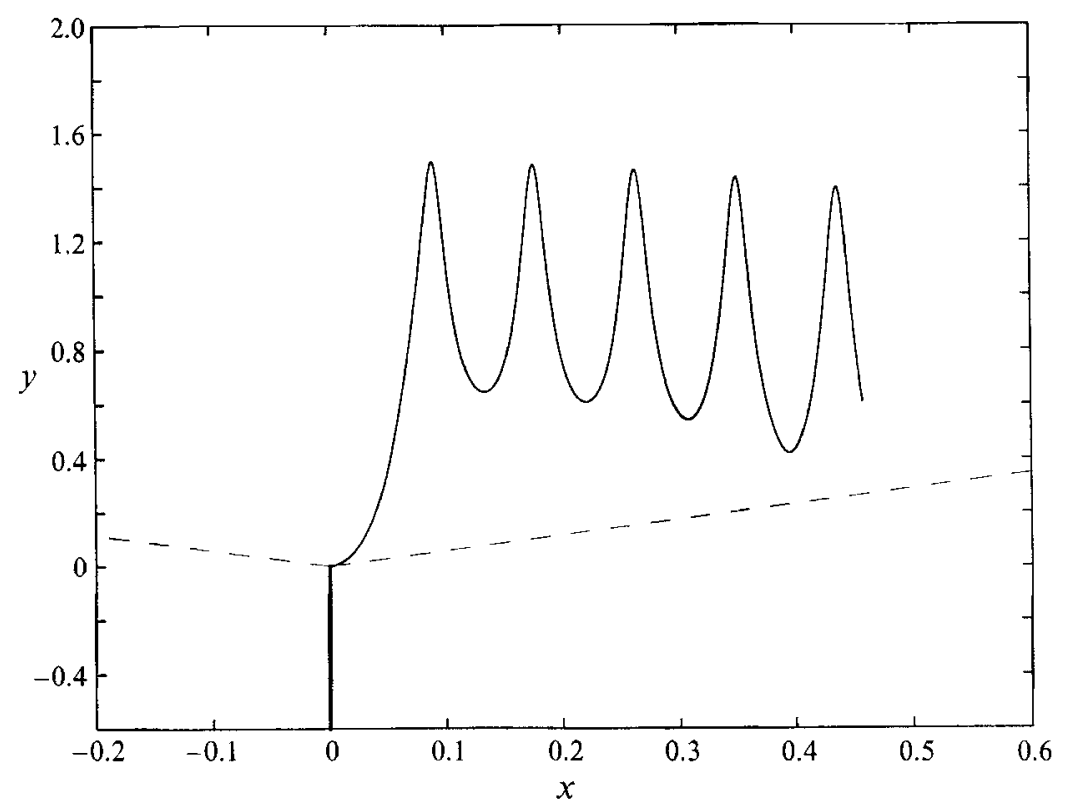

FIGURE 15, Starting vortex trajectory.

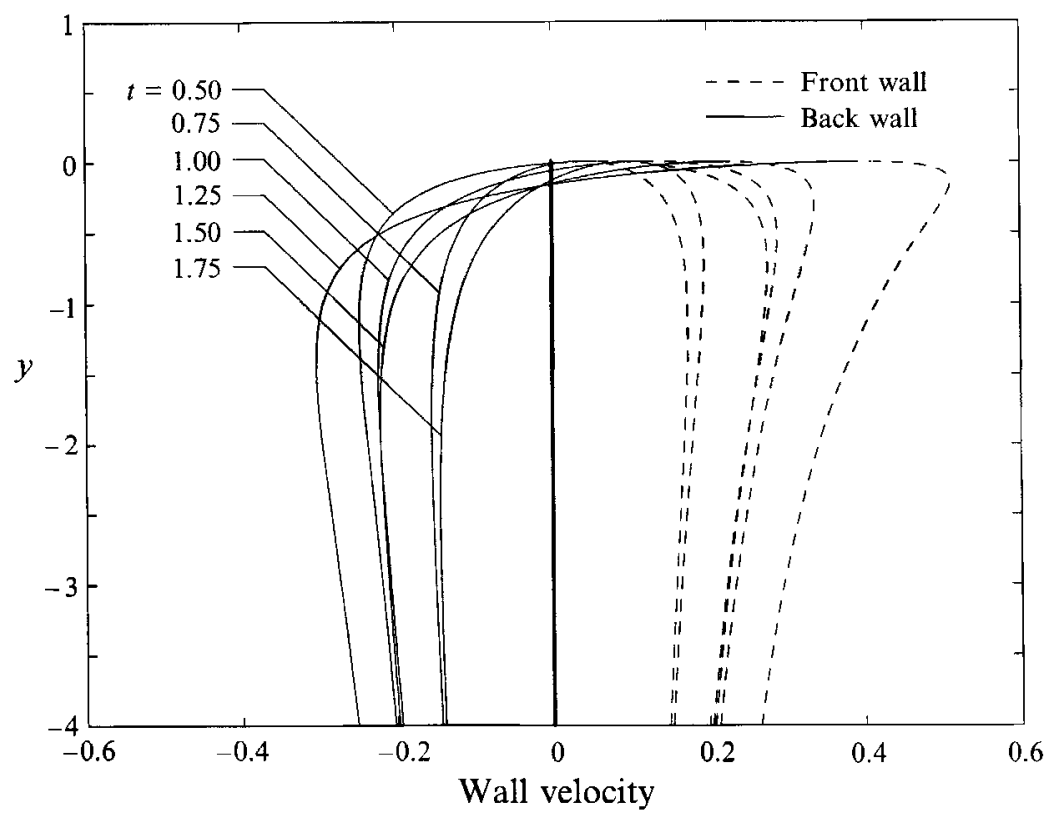

FIGURE 16. Velocity signature on the walls of the plate when $u_{p}$ is subtracted out.

In the second case (vortex in the second sector) these factors become handicaps, the vortex is convected toward the sector divider and its strength is almost half that of the previous case, reducing the controllability of the system. The strength of the vortex is the result of the compromise between having a strong vortex too close to the singularity and a weak one too far away from the tip of the plate. The controller is left with a very delicate task and its performance is not as good as in the first case. 
(a)

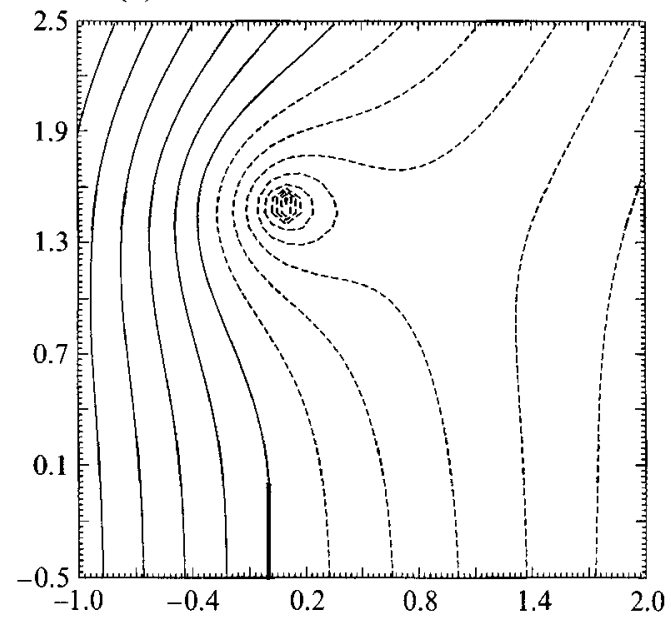

(c)

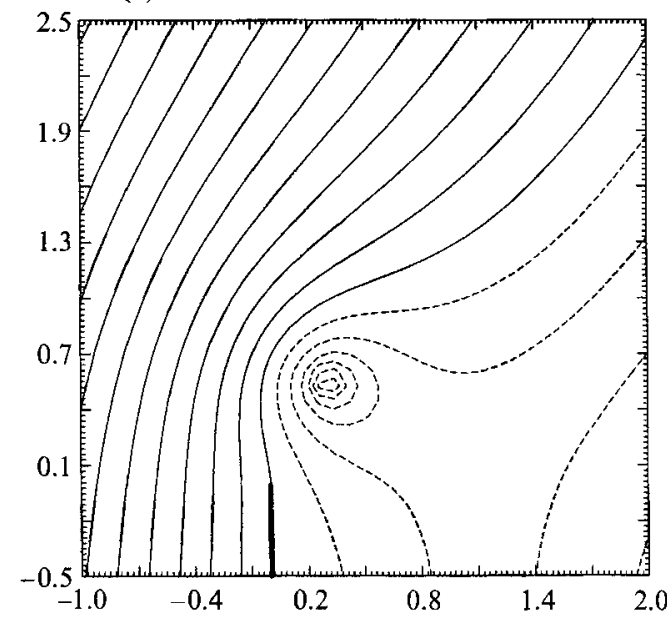

(b)

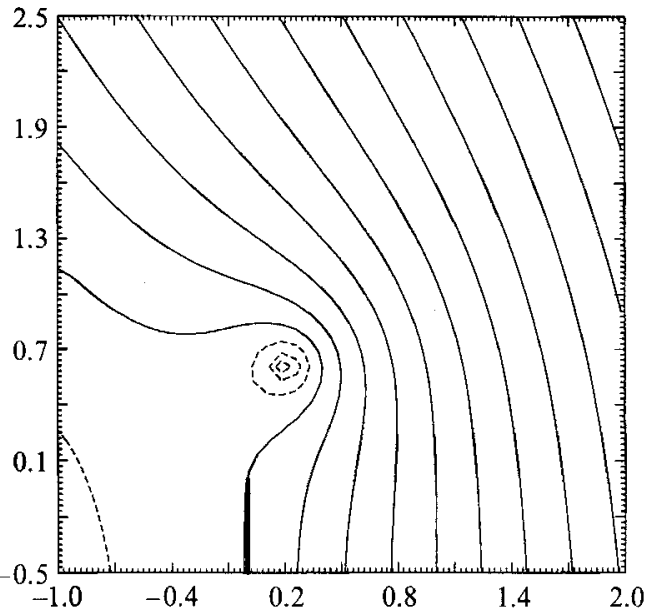

(d)

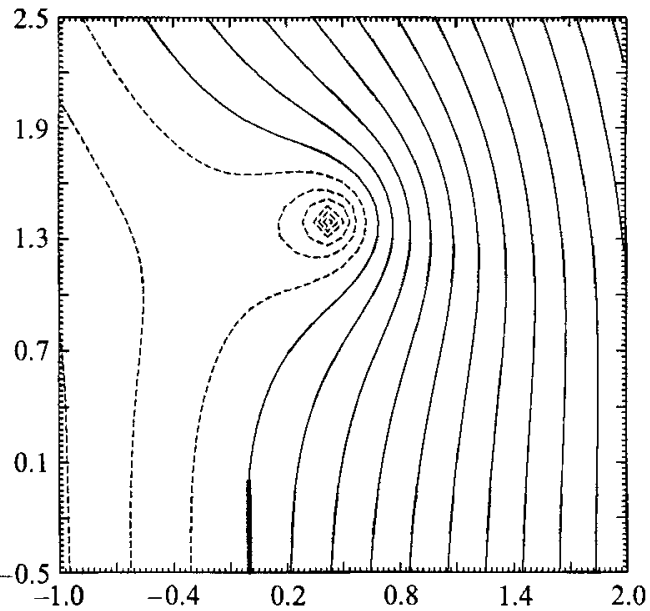

FIGURE 17. Shedding control: (a) $t=0.41,(b) t=0.91,(c) t=1.26,(d) t=1.75$.

\section{Vortex detection}

In $\S 3$ we derived the closed-form solution for the nonlinear controller for the transverse velocity of the plate that inhibits the production of circulation, for any given free-stream condition of definite sign, when the starting vortex is present in the flow. The controller requires, at all time, the free-stream velocity and acceleration and the vortex circulation and position. In a more general situation, where, for example, the controller might be used to control the flow simulated by another technique, it may not be possible or desirable to determine the above information directly but it may be necessary to deduce it from the flow field. The free-stream velocity does not represent a problem because it is known or is easily measurable, but the vortex parameters are not trivial to obtain. In this section we provide a procedure to identify the location and the strength of the vortex, knowing the velocity field near the tip of the plate. We show also that this provides a physical explanation for the controllability regions.

Figures 11 and 16 show that the presence of the vortex produces a well-defined velocity signature on the walls of the plate (above the boundary layer). Within our 
model the velocity field in the physical plane is obtained by multiplying the velocity field in the mapped plane (2) by the Jacobian of the mapping, i.e. by $\mathrm{d} \zeta / \mathrm{d} z$. Then, the mapping could be used to determine the velocity field in terms of physical coordinates. However, because this transformation generates a complicated expression for the wall velocity, we choose to work with the physical velocity written in terms of the mapped coordinates $\zeta=\xi+\mathrm{i} \eta$. The plate walls in the mapped plane are along the real axis $\eta=0$, and the velocity on this axis is given by

$$
u_{u v a l l}=-\frac{U\left(\xi-2 \xi_{1}\right)}{2\left[\xi_{1}^{2}+\eta_{1}^{2}+\xi\left(\xi-2 \xi_{1}\right)\right]}-u_{p}
$$

The velocity at the separation point is obtained by setting $\xi=0$. We note that there is a stagnation point at $\xi=2 \xi_{1}$ when the plate is stationary, while two stagnation points are identifiable if the plate velocity satisfies the following inequality:

$$
\frac{U}{4 \eta_{1}^{2}}\left[\xi_{1}-\operatorname{sgn}(U)\left(\xi_{1}^{2}+\eta_{1}^{2}\right)^{\frac{1}{2}}\right]<u_{p}<\frac{U}{4 \eta_{1}^{2}}\left[\xi_{1}+\operatorname{sgn}(U)\left(\xi_{1}^{2}+\eta_{1}^{2}\right)^{\frac{1}{1}}\right], \quad \forall U .
$$

When the plate velocity is outside these bounds there are no stagnation points, i.e. the zero streamline leaves the tip of the plate and extends to infinity (e.g. see figure 17a-d). To characterize the velocity signature in terms of the vortex location, we compute the derivative of the wall velocity along the $\xi$-axis. We find that the wall velocity has two extrema:

$$
\xi_{l}=2 \xi_{1}-\left(\xi_{1}^{2}+\eta_{1}^{2}\right)^{\frac{1}{2}}, \quad \xi_{r}=2 \xi_{1}+\left(\xi_{1}^{2}+\eta_{1}^{2}\right)^{\frac{1}{2}},
$$

where the subscripts $l$ and $r$ indicate left and right. Note that the locations of the extrema do not depend directly on the free-stream velocity nor on the velocity of the plate but exclusively on the position of the vortex. The magnitudes of the wall velocity at these points are

$$
u_{l}=-\frac{U}{4\left[\xi_{1}-\left(\xi_{1}^{2}+\eta_{1}^{2}\right)^{\frac{1}{2}}\right]}-u_{p}, \quad u_{r}=-\frac{U}{4\left[\xi_{1}+\left(\xi_{1}^{2}+\eta_{1}^{2}\right)^{\frac{1}{2}}\right]}-u_{p} .
$$

Let us restrict our discussion to the case when $U(t)>0$ for all $t>0$, then, from the results presented in the previous sections, it follows that $\xi_{1}(t)>0$ for all $t>0$. In this case it is easy to see that $u_{l}$ is a maximum while $u_{r}$ is a minimum. The minimum is always located on the back face of the plate because $\xi_{r}$ is always positive. It is easy to show that the maximum is located on the front face of the plate if $\eta_{1}<\xi_{1} \sqrt{ } 3$, while it moves on the back face of the plate otherwise. Recalling that $\xi=\rho \sin \theta$ and $\eta=\rho \cos \theta$ (see figure 1) it follows that the maximum can be found on the front face of the plate if $\theta_{1}<\frac{1}{6} \pi$, while is located on the back face otherwise. When $\theta_{1}=\frac{1}{6} \pi$ the maximum velocity is at the separation point, i.e. at the tip of the plate. The argument can be easily reversed to obtain the results when $U(t)<0$. Figures 11 and 16 show that as the vortex moves within a sector the location and the magnitude of the extrema change but the structure of the velocity signature remains the same. Consequently, the lines $\theta= \pm \frac{1}{6} \pi$ gain further importance: the fluid domain is divided in three sectors not only because of controllability reasons, but also because the structure of the flow field depends upon the sector in which the vortex is located. The fact that the flow becomes uncontrollable when the location of the maximum wall velocity coincides with the separation point suggests that knowledge of the velocity field near the tip of the plate might provide insight into the controllability of the system.

If we consider (45) and (46) we can see that it is possible to extract information about the position of the vortex when the location and magnitude of one of the extrema is 
measurable. On the other hand, knowledge of the wall velocity around the tip of the plate is already important for controllability reasons, consequently it might be more reasonable to predict the position and circulation of the vortex from the wall velocity and its derivative at the tip of the plate. Another advantage of this choice is that these quantities are simpler to measure. Using the expression for the wall velocity (43) and computing its derivative with respect to $\xi$ and then evaluating both expressions at the origin we obtain the following equations:

$$
\frac{U \xi_{1}}{\xi_{1}^{2}+\eta_{1}^{2}}=u_{0}, \quad \frac{U\left(3 \xi_{1}^{2}-\eta_{1}^{2}\right)}{2\left(\xi_{1}^{2}+\eta_{1}^{2}\right)^{2}}=u_{0}^{\prime},
$$

where $u_{0}$ is the fluid velocity at the tip of the plate with the plate velocity subtracted out, i.e. $u_{0}=u_{t i p}+u_{p}$, while $u_{0}^{\prime}$ is the spatial derivative of the velocity 'around' the tip of the plate. To compute this derivative we must measure the fluid velocity on the walls of the plate at distance $\frac{1}{2} \Delta y$ from its tip. Then, the measured velocities would be associated with the two corresponding points of the $\zeta$-plane which lie on the $\xi$-axis symmetrically with respect to the origin. If we call $\Delta \xi$ the distance between these two points, then this quantity is related to $\Delta y$ through the mapping (1). Consequently, the derivative 'around' the tip of the plate can be defined as

$$
u_{0}^{\prime}=\lim _{\Delta \xi \rightarrow 0} \frac{u\left(\frac{1}{2} \Delta \xi\right)-u\left(-\frac{1}{2} \Delta \xi\right)}{\Delta \xi} .
$$

Solving the set of equations (47) we obtain

$$
\xi_{1}=\frac{U u_{0}}{2\left(2 u_{0}^{2}-U u_{0}^{\prime}\right)}, \quad \eta_{1}=\frac{U\left(3 u_{0}^{2}-2 U u_{0}^{\prime}\right)^{\frac{1}{2}}}{2\left(2 u_{0}^{2}-U u_{0}^{\prime}\right)},
$$

where $\zeta_{1}=\xi_{1}+i \eta_{1}$ is the predicted position of the vortex. The final step is to estimate the circulation of the vortex. To be consistent with our model the vortex has to satisfy the Kutta condition, hence using the estimate location of the vortex and (3) we obtain

$$
\Gamma_{1}=\frac{\pi U^{2}}{\left(3 u_{0}^{2}-2 U u_{0}^{\prime}\right)^{\frac{1}{2}}} \text {. }
$$

Finally, the predicted position and circulation of the vortex can be fed back to the controller, i.e. we can compute the plate velocity using (22). It is actually possible to save one step in the feedback process by rewriting the controller in terms of $u_{0}$ and $u_{0}^{\prime}$. Substituting (49) and (50) in (22) we obtain

$$
u_{p}=-\frac{1}{u_{0}^{\prime}}\left[\frac{u_{0}\left(2 u_{0}^{2}-U u_{0}^{\prime}\right)\left(6 u_{0}^{2}-5 U u_{0}^{\prime}\right)}{4 U\left(3 u_{0}^{2}-2 U u_{0}^{\prime}\right)^{\frac{1}{2}}}+\frac{\mathrm{d} U}{\mathrm{~d} t}\right] .
$$

This expression is equivalent to (22) - the plate velocity goes to infinity when $u_{0}^{\prime}$ goes to zero, i.e. when the vortex approaches the lines $\theta= \pm \frac{1}{6} \pi$. We want to underline that (49)-(51) are well defined and no extra singularities have been introduced by the above derivation. It is easy to show, using (47), that $2 u_{0}^{2}-U u_{0}^{\prime} \geqslant 0$ and $3 u_{0}^{2}-2 U u_{0}^{\prime} \geqslant 0, \forall U$, $\forall \xi_{1}, \eta_{1}$. Equation (51), therefore, also reflects the other two singularities discussed at the end of $\S 3$, i.e. the plate velocity has to become infinite when $U$ goes to zero or when the vortex approaches the walls of the plate.

Let us conclude this section by rewriting the rate of circulation production (12) in terms of $u_{0}$ and $u_{0}^{\prime}$. We obtain

$$
\frac{\mathrm{d} \Gamma_{1}}{\mathrm{~d} t}=\frac{2 \pi}{3\left(3 u_{0}^{2}-2 U u_{0}^{\prime}\right)^{\frac{1}{2}}}\left[\frac{u_{0}\left(2 u_{0}^{2}-U u_{0}^{\prime}\right)\left(6 u_{0}^{2}-5 U u_{0}^{\prime}\right)}{4\left(3 u_{0}^{2}-2 U u_{0}^{\prime}\right)}+U \frac{\mathrm{d} U}{\mathrm{~d} t}+U u_{0}^{\prime} u_{p}\right] .
$$




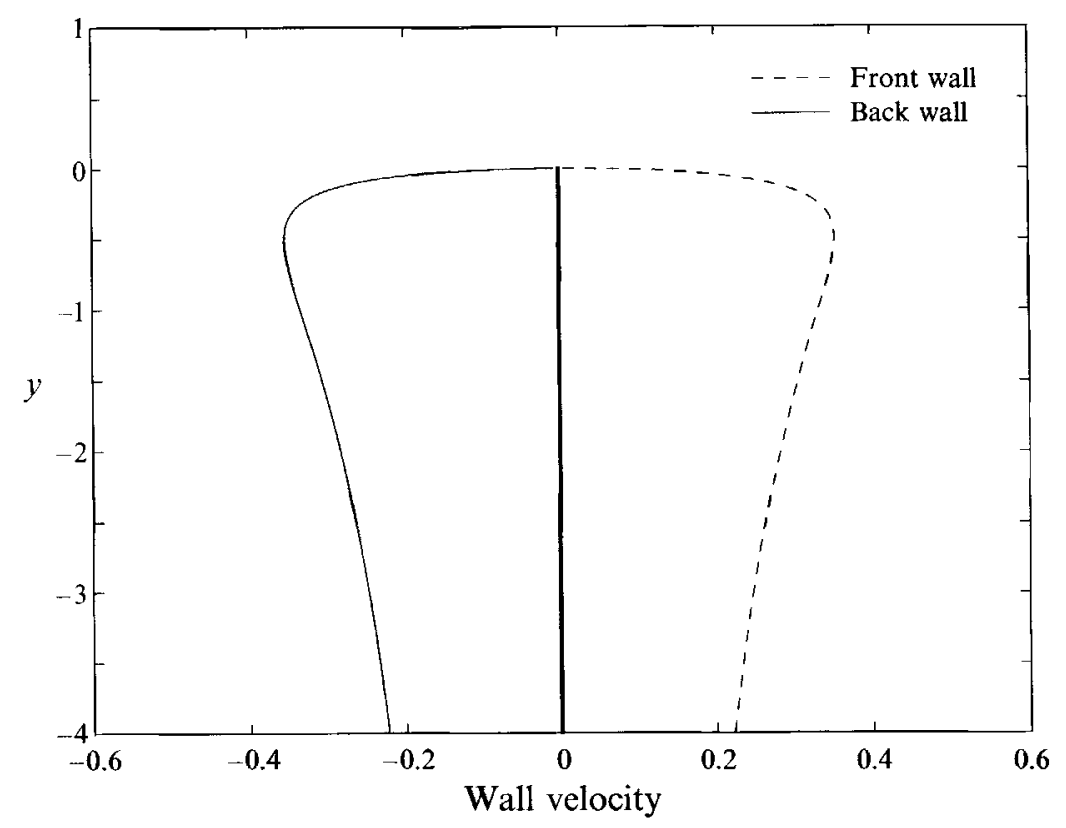

FIGURE 18. Velocity signature on the walls of the plate.

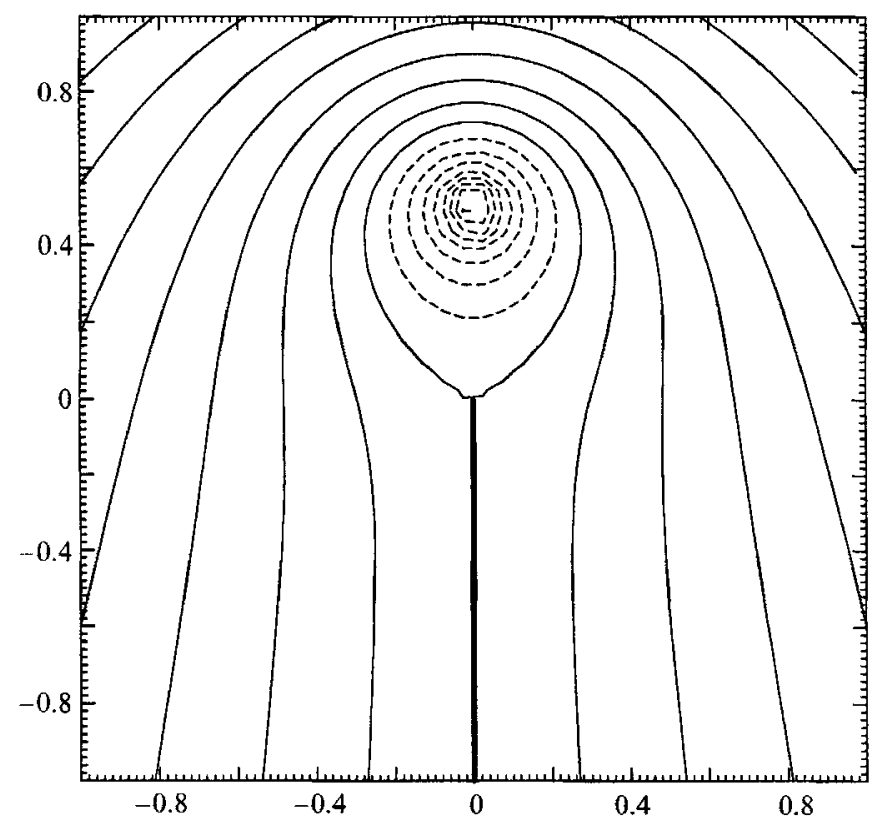

FIGURE 19. Instantaneous streamlines.

This expression and (12) are, of course, equivalent but deeper insight into the flow is obtained only from the simultaneous analysis of these two expressions. The first term in square brackets is the contribution to the rate of circulation production due to the free-stream velocity. This term is, in general, non-zero even if the free-stream acceleration is zero and the plate is stationary. However, there are two exceptions: the 
term vanishes when $u_{0}=0 \Leftrightarrow \sin \theta_{1}=0$ or when $6 u_{0}^{2}-5 U u_{0}^{\prime}=0 \Leftrightarrow 8 \cos ^{2} \theta_{1}-3=0$. The first relationship identifies a particular flow structure, the one where the vortex lies on the positive imaginary axis, see figure 19. The flow in this case is symmetric with respect to the plate and the wall velocity vanishes at the origin; consequently the tip of the plate becomes a stagnation point, see figures 18 and 19. The second relationship identifies two lines, $\theta= \pm 0.29 \pi$. When the vortex moves across one of these lines the contribution to the rate of circulation production due to the free-stream velocity changes sign. One should not be misled by this term. Even assuming that $\mathrm{d} U / \mathrm{d} t=0$ and $u_{p}=0$, the vortex cannot move on one of these lines and at the same time satisfy the Kutta condition without requiring further production of circulation, see (11). Furthermore, it cannot stay on one of these lines because of the proven lack of a fixed point for this flow, see $\S 4$.

The second term between square brackets in (12) and (52) is the contribution to the rate of circulation production due to the free-stream acceleration. Note the strong correlation between these two quantities: circulation must be produced if the freestream velocity is unsteady. Consequently, $\mathrm{d} U / \mathrm{d} t$ is a powerful and robust means of controlling the injection of circulation in the flow, although not necessarily easy or convenient to use. For example, if the goal is to control the vortical structures on top of a delta wing it is probably unrealistic to think of changing the velocity of the aircraft to achieve the result.

Finally, the third term between square brackets in (12) and (52) is the contribution to the rate of circulation production due to the motion of the plate. As we can see, it is possible to use the transverse motion of the plate to control the production of circulation only if there is a non-zero gradient in the velocity field at the tip of the plate. As the magnitude of the gradient decreases, increasingly higher plate velocities are necessary to achieve control, and the control is totally lost when the gradient vanishes, i.e. when the vortex reaches $\theta= \pm \frac{1}{6} \pi$. The wall velocity for this limiting case yields a cusp-like maximum at the tip of the plate, see figure 20. Figure 21 shows the associated instantaneous streamlines. It is interesting to observe that when $\theta_{1} \in\left(0, \frac{1}{6} \pi\right)$ the zero streamline, the one leaving the tip of the plate, at first bends upstream while, when $\theta_{1} \in\left(\frac{1}{6} \pi, \frac{1}{2} \pi\right)$ it turns immediately downstream. The case $\theta_{1}= \pm \frac{1}{6} \pi$ constitutes, hence, the borderline between these two situations. This observation suggests that the control is lost when the velocity field around the tip of the plate becomes uniform. To check this hypothesis we expand the complex velocity field about the tip of the plate:

$$
w=\mathrm{i}\left[u_{p}-\frac{U \xi_{1}}{\xi_{1}^{2}+\eta_{1}^{2}}\right]+\mathrm{i} \frac{U(\xi+\mathrm{i} \eta)\left(\eta_{1}^{2}-3 \xi_{1}^{2}\right)}{2\left(\xi_{1}^{2}+\eta_{1}^{2}\right)^{2}}+O(\xi+\mathrm{i} \eta)^{2} .
$$

The leading order is the fluid velocity at the separation point. The first order shows that the velocity field around the tip of the plate in general depends linearly on $\zeta$. Such a dependency is lost when $\eta_{1}= \pm \xi_{1} \sqrt{ } 3$, i.e. when $\theta_{1}= \pm \frac{1}{6} \pi$, and the flow becomes uniform around the tip of the plate. A physical interpretation of the control mechanism is the following: if there is a velocity gradient around the separation point then the motion of the plate changes the flow balance between the two sides of its tip, affecting the production of circulation as necessary to control the flow. On the other hand, when the vortex reaches one of the lines $\theta= \pm \frac{1}{6} \pi$ the velocity field is locally uniform around the tip of the plate and parallel to the plate, consequently a displacement of the plate cannot modify the velocity field around the separation point and ultimately cannot affect the production of circulation. 


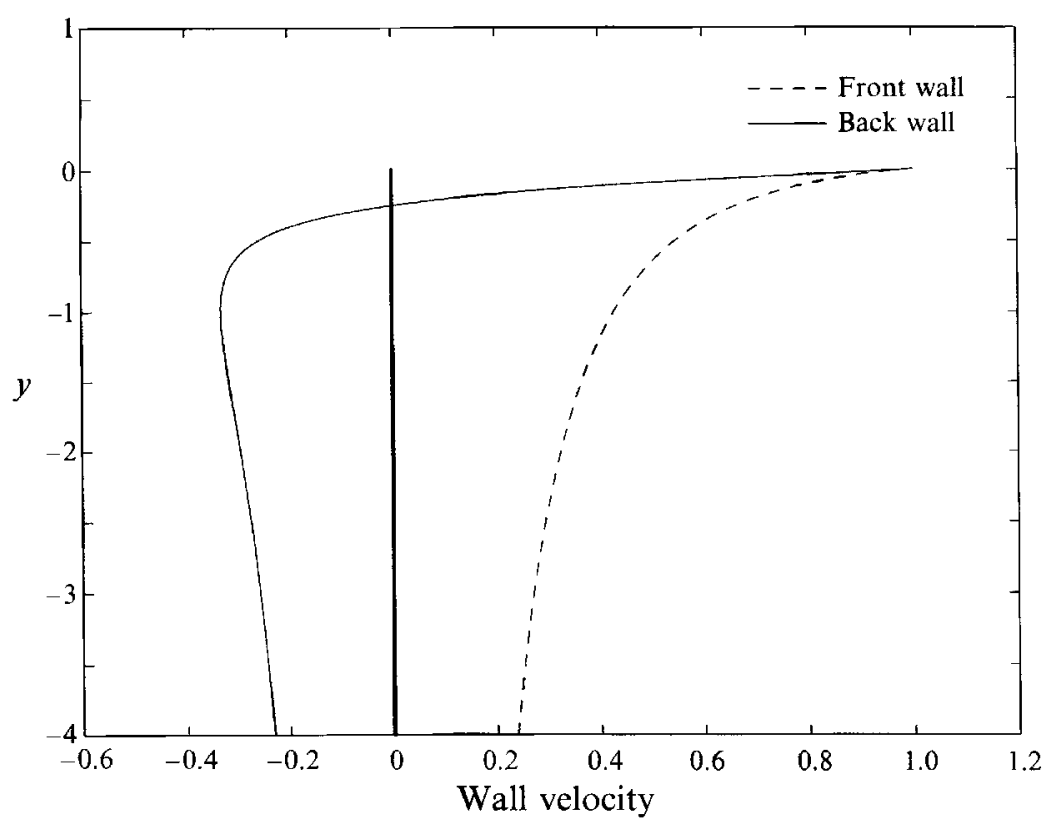

FIGURE 20. Velocity signature on the walls of the plate.

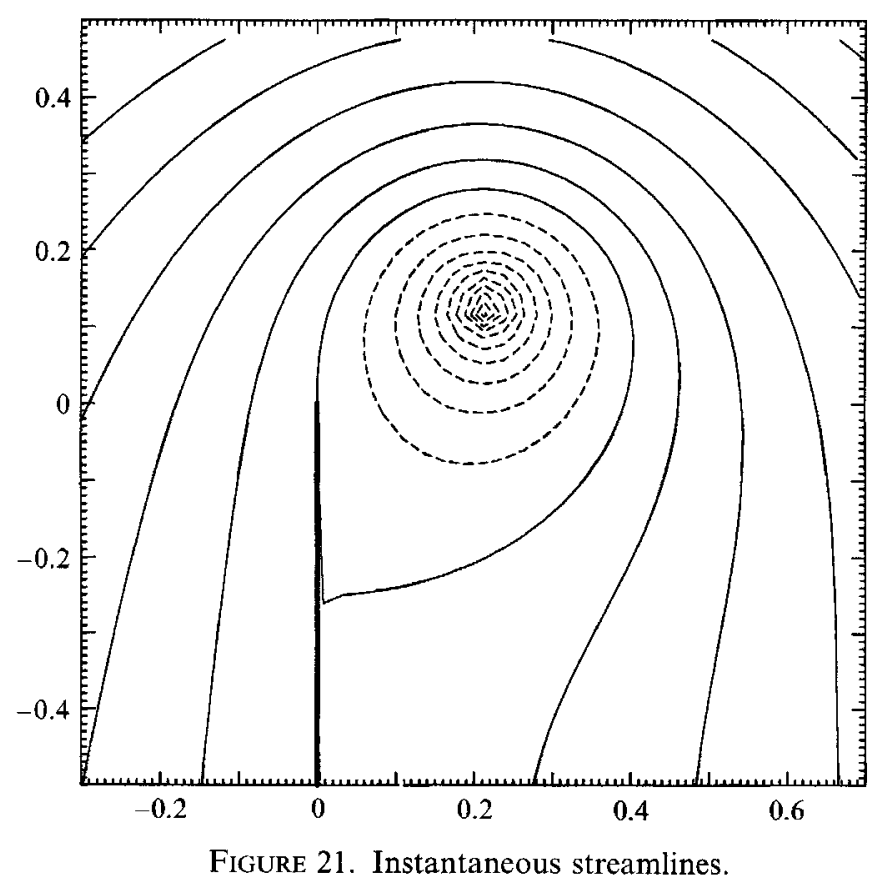

\section{Conclusions}

A point-vortex model has been used to simulate the unsteady separated flow past a semi-infinite plate with transverse motion. Within this model we derived a control strategy that inhibits the production of additional circulation when a vortex is present in the flow. Because of the simplicity of the model we obtained the analytical closed- 
form solution of the controller for any time-dependent free-stream velocity of definite sign. The mathematical derivation of the controller showed that the fluid domain is divided into three controllability regions. The performance of the controller within each region was then characterized with a dynamical systems type of analysis. Using a Liapunov type of argument we proved that the vortex moves irreversibly downstream regardless of the control strategy, i.e. the motion of the plate cannot reverse the trajectory of the vortex. Furthermore, for an oscillatory free stream the analysis of the phase space showed that the vortex is attracted, on average, by the lines $\theta= \pm \frac{1}{6} \pi$. These lines, in the frame of reference fixed with the plate, separate the controllability regions. When the vortex reaches one of these lines the flow becomes uncontrollable because the motion of the plate cannot affect the rate of circulation production. As a consequence of this result the time over which the system is controllable is finite but it can be maximized by an intelligent choice of the shedding conditions. We successfully tested the controller and verified the predictions about its performance when the freestream oscillates about a non-zero mean and showed that the motion of the plate, before the shedding time, can initiate the controlled system in two totally different controllable states. Finally, we presented a procedure to derive, from the velocity signature on the plate, some of the information necessary for feedback to the controller, i.e. the position and the circulation of the vortex. Such information may be deduced from the velocity at the walls near the tip of the plate. We showed that structure of the signature depends on the position of the vortex and we identified three sub-domains, which coincide with the controllability regions, within which the vortex generates the same kind of signature. Furthermore, we proved that the controller is able to modify the rate of circulation production because the motion of the plate changes the flow balance between the two sides of its tip. On the other hand, the control is lost when the vortex reaches the border of the controllability region because the velocity field near the tip of the plate becomes uniform. We conclude that there is a strong correlation between controllability and flow structure and a deep understanding of the fluid flow is required to explain the performance and limitations of the control strategy.

The logical continuation of the present work would be to embed the derived controller into a more realistic CFD code which can, for example, integrate the full Navier-Stokes equation. The possibility of testing the controller in a numerical environment instead of a real experiment is of great utility. On one hand it permits the synchronization of the flow and the controller and on the other it produces information on how the controller performs with a flow model different from the one in which it was derived. Without the difficulties of controlling a laboratory experiment in real time attention can be focused on making the controller progressively more robust with respect to different types of perturbations (e.g. viscosity, three-dimensional, background noise, etc.). Successful iterations of this process might open the possibilities for the active control of large-scale coherent vortical structures in an experiment.

The first author is deeply grateful to Dr R. Camassa and Dr D. D. Holm for several valuable discussions and to the Center for Nonlinear Studies at the Los Alamos National Laboratories for the financial support provided during the summer of 1991. This work was supported by the Office for the Naval Research Grants no. N00014-90J-1589 and no. N00014-89-J-1678 and by Rockwell International. 


\section{REFERENCES}

Brown, C. E. \& MichaEL, W. H. 1954 Effect of leading-edge separation on the lift of a delta wing. J. Aero. Sci. 21, 690-694.

Clements, R. R. 1973 An inviscid model of two-dimensional vortex shedding. J. Fluid Mech. 57, $321-336$.

CortelezzI, L. 1993 Power-law starting flow past a flat plate: scaling and universality. Phys. Fluids (submitted).

Cortelezzi, L. \& LeOnard, A. 1993 Point vortex model for the unsteady separated flow past a semi-infinite plate with transverse motion. Fluid Dyn. Res. 11, 263-295.

Doyle, J. C., Francis, B. A. \& Tannenbaum, A. R. 1992 Feedback Control Theory. Macmillan.

Fan, M. K. H., TITS, A. L. \& Doyle, J. C. 1991 Robustness in the presence of mixed parametric uncertainty and unmodeled dynamics. IEEE Auto. Control 36, 25-38.

GAD-el-HAK, M. \& Bushnell, D. M. 1991 Separation control: review. Trans. ASME I: J. Fluids Engng 113, 5-30.

Graham, J. M. R. 1980 The forces on the sharp-edged cylinders in oscillatory flow at low Keulegan-Carpenter numbers. J. Fluid Mech. 97, 331-346.

Koochesfahani, M. M. \& Dimotakis, P. E. 1988 A cancellation experiment in a forced turbulent shear layer. First Natl Fhid Dyn. Congr., July 25-28, 1988, Cincinnati, Ohio. AIAA Paper 883713-CP.

ONGOREn, A. \& Rockwell, D. 1988 a Flow structure from an oscillating cylinder. Part 1. Mechanisms of phase shift and recovery in the near wake. J. Fluid Mech. 191, 197-223.

ONGOREN, A. \& Rockwell, D. $1988 b$ Flow structure from an oscillating cylinder. Part 2. Mode competition in the near wake. J. Fluid Mech. 191, 225-245.

Pullin, D. I. 1978 The large-scale structure of unsteady self-similar rolled-up vortex sheets. J. Fluid Mech. 88, 401-430.

Rao, D. M. 1987 Vortical flow management techniques. Prog. Aerospace Sci. 24, 173-224.

Slomski, J. F. \& Coleman, R. M. 1993 Numerical simulation of vortex generation and capture above an airfoil. 31st Aerospace Sci. Meeting and Exhibit, January 11-14, 1993, Reno, Nevada. AIAA Paper 93-864.

Tokumaru, P. T. \& Dimotakis, P. E. 1991 Rotary oscillation control of a cylinder wake. J. Fluid Mech. 224, 77-90.

Wiggins, S. 1990 Introduction to Applied Nonlinear Dynamical Systems and Chaos. Springer. 